\title{
Uniform stability of nonlinear time-varying impulsive systems with eventually uniformly bounded impulse frequency
}

\author{
José L. Mancilla-Aguilar ${ }^{\mathrm{a}}$, Hernan Haimovich ${ }^{\mathrm{b}, *}$, Petro Feketa ${ }^{\mathrm{c}}$ \\ anstituto Tecnológico de Buenos Aires, Av. E. Madero 399, Buenos Aires, Argentina. \\ ${ }^{b}$ International Center for Information and Systems Science (CIFASIS), CONICET-UNR, Ocampo y Esmeralda, 2000 \\ Rosario, Argentina. \\ ${ }^{c}$ Kiel University, Chair of Automatic Control, Kaiserstraße 2, 24143 Kiel, Germany
}

\begin{abstract}
We provide novel sufficient conditions for stability of nonlinear and time-varying impulsive systems. These conditions generalize, extend, and strengthen many existing results. Different types of input-to-state stability (ISS), as well as zero-input global uniform asymptotic stability (0-GUAS), are covered by employing a twomeasure framework and considering stability of both weak (decay depends only on elapsed time) and strong (decay depends on elapsed time and the number of impulses) flavors. By contrast to many existing results, the stability state bounds imposed are uniform with respect to initial time and also with respect to classes of impulse-time sequences where the impulse frequency is eventually uniformly bounded. We show that the considered classes of impulse-time sequences are substantially broader than other previously considered classes, such as those having fixed or (reverse) average dwell times, or impulse frequency achieving uniform convergence to a limit (superior or inferior). Moreover, our sufficient conditions are not more restrictive than existing ones when particularized to some of the cases covered in the literature, and hence in these cases our results allow to strengthen the existing conclusions.
\end{abstract}

Keywords: Impulsive systems, nonlinear systems, time-varying systems, input-to-state stability, hybrid systems.

\section{Introduction}

The theory of impulsive systems (Samoilenko and Perestyuk, 1987; Lakshmikantham et al., 1989) is a convenient mathematical framework for modeling processes that combine continuous and discontinuous behaviors. An impulsive system consists of an ordinary differential equation that governs the evolution of the state between jumps, a static law which introduces discontinuities at some isolated moments of time, and an impulse-time sequence which determines the instants when the static law comes into play. Applications of impulsive systems can be found in robotics (Tang et al., 2015), biomedical engineering (Rivadeneira and Moog, 2015), population dynamics (Rogovchenko, 1997; Yang et al., 2019), and many other areas. The basis of the mathematical theory of impulsive systems as well as fundamental results on the existence and local stability of solutions are summarized in the monographs by Samoilenko and Perestyuk (1987); Lakshmikantham et al. (1989); Samoilenko and Perestyuk (1995).

Many recently developed methods for the global stability analysis of impulsive systems are tightly related to the notion of input-to-state stability (ISS). ISS was introduced by Sontag (1989) for continuous-time systems with inputs and characterizes the behavior of solutions with respect to external disturbances. ISS of

\footnotetext{
${ }^{*}$ Corresponding author

Email addresses: jmancill@itba.edu.ar (José L. Mancilla-Aguilar), haimovich@cifasis-conicet.gov.ar (Hernan Haimovich), pf@tf.uni-kiel.de (Petro Feketa)
} 
impulsive control systems was firstly studied in Hespanha et al. $(2005,2008)$ by providing a set of Lyapunovbased sufficient conditions that ensure ISS with respect to suitable classes of impulse-time sequences. These results were obtained by introducing the concept of exponential ISS-Lyapunov function. Two constants, called rate coefficients, are used to bound the evolution of the ISS-Lyapunov function along the trajectories of impulsive system during flows (constant $c \in \mathbb{R}$ ) and jumps (constant $d \in \mathbb{R}$ ). Relations called dwelltime conditions (DTC) which restrict the number/frequency of jumps in order to guarantee ISS have been introduced. These conditions can be of two types: fixed or average dwell-time. The fixed dwell-time conditions utilize the minimum/maximum time-distance between two consecutive jumps. The average ones impose a bound for the number of jumps in some average sense. Sufficient conditions for ISS which are based on fixed dwell-time conditions are less widely applicable than the ones based on average dwell-time conditions. Some generalizations of the recently discussed approach, involving exponential ISS-Lyapunov functions with multiple and time-varying rate coefficients, have been proposed in Dashkovskiv and Feketa (2017, 2016) and Peng et al. (2018); Peng (2018); Ning et al. (2018), respectively. In addition, the ideas of Hespanha et al. (2005, 2008) have been extended to some classes of time-delay (Dashkovskiv et al., 2012; Liu et al., 2011; Sun and Wang, 2012; Wu et al., 2016), switched (Liu et al., 2011, 2012), and stochastic (Ren and Xiong, 2017; Yao et al., 2014; Wu et al., 2016) impulsive systems.

A more refined technique for the ISS and global stability analysis of impulsive control systems that relies on the concept of a candidate Lyapunov function with nonlinear rate functions has been employed in Liu et al. (2012); Dashkovskiy and Mironchenko (2013); Feketa and Bajcinca (2018, 2019a, b); ; Mancilla-Aguilar and Haimo (2019). The nonlinear rate functions are used to bound the evolution of the candidate Lyapunov function along the nonlinear flows and jumps of the impulsive system more precisely than what is possible by means of an exponential-type Lyapunov function (Hespanha et al., 2005, 2008). Hence, the corresponding sufficient conditions are supposed to be less conservative. A drawback of most sufficient conditions involving candidate Lyapunov functions with nonlinear rates was that these conditions are valid over impulse-time sequences having fixed dwell times. This may reduce applicability and thus degrade the benefits of employing nonlinear rates. To the best of authors' knowledge, the recent papers Feketa and Baicinca (2019a, $\mathrm{b}$ ) are the only works that provide sufficient conditions for the (nonuniform) ISS and global asymptotic stability (GAS) of nonlinear impulsive systems in terms of candidate Lyapunov functions with nonlinear rates over impulse-time sequences having average-type dwell-time bounds.

The majority of results on stability of impulsive systems consider a bound on the state that decays as time elapses but is insensitive to the occurrence of jumps. In a time-varying setting, this stability notion is not robust and too weak to be meaningful, as shown in Haimovich and Mancilla-Aguilar (2019a). By contrast, a stronger stability concept where the bound on the state also decays when jumps occur, as usually considered for hybrid systems (Cai and Teel, 2009), indeed is robust and more meaningful for impulsive systems in a time-varying setting (Haimovich and Mancilla-Aguilar, 2019b).

Motivated by Feketa and Baicinca (2019a, $\mathrm{b})$, the contribution of this paper arises from the combination and improvement of the benefits of average-type dwell-time bounds and the most widely applicable uniform ISS results of Mancilla-Aguilar and Haimovich (2019). First, we significantly broaden the class of impulsetime sequences over which our stability results hold by considering impulse-time sequences having eventually uniformly bounded impulse frequency (see Section 3). We show that many known classes of impulse-time sequences are uniform subsets (see Definition 3.3) of the newly proposed classes (Lemma 3.6). Then, we provide sufficient conditions for both weak and strong ISS of impulsive systems where the ISS bounds hold uniformly over initial time and over classes of impulse-time sequences. These conditions are based on Lyapunov-type functions having nonlinear rates. For increased generality, we formulate our results in a (time-varying) two-measure framework that incorporates different important stability notions unifyingly. Our results are thus stronger, less conservative and more widely applicable than many existing results.

The remainder of the paper is organized as follows. In Section 2 we introduce notation, the type of systems and the stability definitions considered. In Section 3, we introduce different classes of impulse-time sequences and show the relationships between these and existing classes. The main results of the paper, consisting in most general Lyapunov-based sufficient conditions for stability, uniformly with respect to both initial time and impulse-time sequences within the considered classes, are provided in Section 4. Application examples are provided in Section [5 and conclusions in Section [6 


\section{Basic definitions}

\subsection{Notation}

The reals, positive, and nonnegative reals are denoted by $\mathbb{R}, \mathbb{R}_{>0}$ and $\mathbb{R}_{>0}$, respectively. A function $\varphi: \mathbb{R}_{\geq 0} \rightarrow \mathbb{R}_{\geq 0}$ is said to be of class $\mathcal{C}_{*}$, written $\varphi \in \mathcal{C}_{*}$, if $\varphi$ is continuous and satisfies $\varphi(0)=0$. If $\varphi \in \mathcal{C}_{*}$, we write $\varphi \in \mathcal{P}$ if $\varphi(s)>0$ for all $s>0 ; \varphi \in \mathcal{K}$ if $\varphi$ is strictly increasing; and $\varphi \in \mathcal{K}_{\infty}$ if $\varphi \in \mathcal{K}$ and $\varphi$ is unbounded. It is clear that $\mathcal{K}_{\infty} \subset \mathcal{K} \subset \mathcal{P} \subset \mathcal{C}_{*}$. If $\beta: \mathbb{R}_{\geq 0} \times \mathbb{R}_{\geq 0} \rightarrow \mathbb{R}_{\geq 0}$ is continuous, we write $\beta \in \mathcal{K} \mathcal{L}$ if $\beta(\cdot, t) \in \mathcal{K}$ for all $t \geq 0, \beta(r, \cdot)$ is strictly decreasing whenever positive, and $\lim _{t \rightarrow \infty} \beta(r, t)=0$ for all $r \geq 0$. For $a \in \mathbb{R},\lfloor a\rfloor$ and $\lceil a\rceil$ denote, respectively, the greatest integer not greater than $a$ and the least integer not less than $a$. For any function $x: I \subset \mathbb{R} \rightarrow \mathbb{R}^{n}$, with $I$ an open interval, $x\left(t^{-}\right)$and $x\left(t^{+}\right)$denote respectively the left and right limits of $x$ at $t \in I$. For an infinite sequence $\left\{\Delta_{k}\right\}_{k=1}^{\infty}$ of real numbers, $\Delta_{k} \nearrow L$ means that the sequence is strictly increasing and that $\lim _{k \rightarrow \infty} \Delta_{k}=L$.

\subsection{The system}

Consider the time-varying impulsive system with inputs defined by the equations

$$
\begin{array}{ll}
\dot{x}(t)=f(t, x(t), u(t)), & \text { for } t \notin \gamma, \\
x(t)=x\left(t^{-}\right)+g\left(t, x\left(t^{-}\right), u(t)\right), & \text { for } t \in \gamma,
\end{array}
$$

where $t \geq 0$, the state variable $x(t) \in \mathbb{R}^{n}$, the input variable $u(t) \in \mathbb{R}^{m}, f$ (the flow map) and $g$ (the jump map) are functions from $\mathbb{R}_{\geq 0} \times \mathbb{R}^{n} \times \mathbb{R}^{m}$ to $\mathbb{R}^{n}$, and $\gamma=\left\{\tau_{k}\right\}_{k=1}^{N} \subset(0, \infty)$, with $N$ finite or $N=\infty$ is the impulse-time sequence. By "input", we mean a Lebesgue measurable and locally essentially bounded function $u:[0, \infty) \rightarrow \mathbb{R}^{m}$; we denote by $\mathcal{U}$ the set of all the inputs. We only consider impulse-time sequences $\gamma=\left\{\tau_{k}\right\}_{k=1}^{N}$ that are strictly increasing and have no finite limit points, i.e. $\lim _{k \rightarrow \infty} \tau_{k}=\infty$ when the sequence is infinite; we employ $\Gamma$ to denote the set of all such impulse-time sequences. For any sequence $\gamma=\left\{\tau_{k}\right\}_{k=1}^{N} \in \Gamma$ we define for convenience $\tau_{0}=0$ and $\tau_{N+1}=\infty$ when $N$ is finite; nevertheless, $\tau_{0}$ is never an impulse time, because $\gamma \subset(0, \infty)$ by definition.

We assume that for each input $u \in \mathcal{U}$ the map $f_{u}(t, \xi):=f(t, \xi, u(t))$ is a Carathéodory function. Hence the (local) existence of solutions of the differential equation $\dot{x}(t)=f(t, x(t), u(t)$ ) is ensured (see Hale, 1980, Thm. I.5.1).

A solution of (11) corresponding to initial time $t_{0} \geq 0$, initial state $x_{0} \in \mathbb{R}^{n}$, input $u \in \mathcal{U}$ and impulse-time sequence $\gamma$ is a function $x:\left[t_{0}, T_{x}\right) \rightarrow \mathbb{R}^{n}$ such that:

a) $x\left(t_{0}\right)=x_{0}$;

b) $x$ is locally absolutely continuous on each nonempty interval of the form $J_{k}=\left[\tau_{k}, \tau_{k+1}\right) \cap\left[t_{0}, T_{x}\right)$ and $\dot{x}(t)=f(t, x(t), u(t))$ for almost all $t \in J_{k} ;$ and

c) for all $\tau_{k} \in\left(t_{0}, T_{x}\right)$, the left limit $x\left(\tau_{k}^{-}\right)$exists and is finite, and it happens that

$$
x\left(\tau_{k}\right)=x\left(\tau_{k}^{-}\right)+g\left(\tau_{k}, x\left(\tau_{k}^{-}\right), u\left(\tau_{k}\right)\right) .
$$

Note that $\mathrm{b}$ ) implies that for all $t \in\left[t_{0}, T_{x}\right), x(t)=x\left(t^{+}\right)$, i.e. $x$ is right-continuous at $t$.

The solution $x$ is said to be maximally defined if no other solution $y:\left[t_{0}, T_{y}\right) \rightarrow \mathbb{R}^{n}$ satisfies $y(t)=x(t)$ for all $t \in\left[t_{0}, T_{x}\right)$ and has $T_{y}>T_{x}$. We use $\mathcal{T}\left(t_{0}, x_{0}, u, \gamma\right)$ to denote the set of maximally defined solutions of (11) corresponding to initial time $t_{0}$, initial state $x_{0}$, input $u$ and impulse-time sequence $\gamma$. Since solutions locally exist but are not necessarily unique, the set $\mathcal{T}\left(t_{0}, x_{0}, u, \gamma\right)$ is nonempty but may contain more than one solution.

Note that even if $t_{0} \in \gamma$, any solution $x \in \mathcal{T}\left(t_{0}, x_{0}, u, \gamma\right)$ begins its evolution by "flowing" and not by "jumping". This is because in item (c) above, the time instants where jumps occur are those in $\gamma \cap\left(t_{0}, T_{x}\right)$. 


\subsection{Input-to-state stability (ISS)}

From (1b), it is clear that the input values at impulse instants may instantaneously affect the state trajectory. Suitable stability properties must hence take the latter fact into consideration. For a given input $u \in \mathcal{U}$ and impulse-time sequence $\gamma \in \Gamma$, we thus consider the following bound over an interval $I \subset \mathbb{R}_{\geq 0}$ :

$$
\left\|u_{I}\right\|_{\gamma}:=\max \left\{\operatorname{ess}_{\left.\sup _{t \in I}|u(t)|, \sup _{t \in \gamma \cap I}|u(t)|\right\} .}\right.
$$

This definition is in agreement with that employed in Cai and Teel (2005, 2009) in the context of hybrid systems.

For greater generality, we formulate the stability properties in the framework of two measures (see Liu et al., 2012; Chatterjee and Liberzon, 2006). Let $\mathcal{H}$ be the set of functions $h: \mathbb{R}_{\geq 0} \times \mathbb{R}^{n} \rightarrow \mathbb{R}_{\geq 0}$. For $\gamma \in \Gamma$ and $t>s \geq 0$, let $n_{(s, t]}^{\gamma}$ be the number of impulse-time instants contained in $(s, t]$, that is

$$
n_{(s, t]}^{\gamma}:=\#[\gamma \cap(s, t]] .
$$

Definition 2.1. Let $h^{\circ}, h \in \mathcal{H}$ and $\mathcal{S} \subset \Gamma$. We say that the impulsive system (1) is

- weakly $\left(h^{o}, h\right)$-ISS over $\mathcal{S}$ if there exist $\beta \in \mathcal{K} \mathcal{L}$ and $\rho \in \mathcal{K}_{\infty}$ such that for all $t_{0} \geq 0, x_{0} \in \mathbb{R}^{n}, u \in \mathcal{U}$, $\gamma \in \mathcal{S}$ and $x \in \mathcal{T}\left(t_{0}, x_{0}, u, \gamma\right)$, it happens that for all $t \in\left[t_{0}, T_{x}\right)$,

$$
h(t, x(t)) \leq \beta\left(h^{o}\left(t_{0}, x_{0}\right), t-t_{0}\right)+\rho\left(\left\|u_{\left(t_{0}, t\right]}\right\|_{\gamma}\right) ;
$$

- strongly $\left(h^{o}, h\right)$-ISS over $\mathcal{S}$ if there exist $\beta \in \mathcal{K} \mathcal{L}$ and $\rho \in \mathcal{K}_{\infty}$ such that for all $t_{0} \geq 0, x_{0} \in \mathbb{R}^{n}, u \in \mathcal{U}$, $\gamma \in \mathcal{S}$ and $x \in \mathcal{T}\left(t_{0}, x_{0}, u, \gamma\right)$, it happens that for all $t \in\left[t_{0}, T_{x}\right)$,

$$
h(t, x(t)) \leq \beta\left(h^{o}\left(t_{0}, x_{0}\right), t-t_{0}+n_{(s, t]}^{\gamma}\right)+\rho\left(\left\|u_{\left(t_{0}, t\right]}\right\|_{\gamma}\right) .
$$

By suitable selection of $h^{0}$ and $h$, one can recover the definitions of different stability properties usually considered in the analysis of both impulsive and nonimpulsive systems. For example, with $h^{0}(t, x)=$ $h(t, x)=|x|$, the weak $\left(h^{o}, h\right)$-ISS property becomes the standard ISS property considered in the literature of systems with inputs. By considering, in addition, that the set where the inputs take values is $\mathbb{R}^{0}:=\{0\}$, then the standard definition of global uniform asymptotic stability (GUAS) for systems without inputs is recovered. By taking $h^{0}(t, x)=|x|$ we obtain an extension of the input-to-output stability property (IOS) studied in Sontag and Wang (2000); see Liu et al. (2012) for more examples.

The decaying term in a weak property is insensitive to jumps, whereas that of a strong property forces additional decay whenever a jump occurs. The weak ISS property is the one considered in most of the literature on impulsive systems with inputs, whereas strong ISS is in agreement with the ISS property for hybrid systems as in Liberzon et al. (2014). The weak stability properties are, however, not robust in the context of time-varying systems (see Haimovich and Mancilla-Aguilar, 2019a).

\section{Classes of impulse-time sequences}

Our main interest is to provide stability results that hold uniformly over both initial time and broad classes of impulse-time sequences. To this aim, we consider several classes of sequences involving upper bounds (Section 3.1) and lower bounds (Section 3.2) on the number of impulses. In Section 3.4, we provide preliminary results establishing properties and relationships between the classes. 


\subsection{Classes involving upper bounds}

Definition 3.1. A family of impulse-time sequences $\mathcal{S} \subset \Gamma$ is said to be uniformly incrementally bounded (UIB, Haimovich and Mancilla-Aquilan, 2018) if there exists a continuous and nondecreasing function $\phi$ : $\mathbb{R}_{>0} \rightarrow \mathbb{R}_{\geq 0}$ such that $n_{(s, t]}^{\gamma} \leq \phi(t-s)$ for all $t>s \geq 0$, and all $\gamma \in \mathcal{S}$.

Every sequence contained in some UIB family $\mathcal{S}$ is such that the number of impulses occuring in a period of fixed finite duration cannot become infinite as the initial time for such a period becomes increasingly large. A specific subfamily of the UIB class is that of average dwell-time sequences with chatter bound $\mathrm{n} \in \mathbb{N}$ and average dwell time $\tau>0$, defined as

$$
\mathcal{S}_{\mathrm{AD}}(\mathrm{n}, \tau)=\left\{\gamma \in \Gamma: n_{(s, t]}^{\gamma} \leq \mathrm{n}+\frac{t-s}{\tau}, \forall 0 \leq s \leq t\right\} .
$$

It is clear that $\mathcal{S}_{\mathrm{AD}}(\mathrm{n}, \tau)$ is UIB for every $\mathrm{n} \in \mathbb{N}$ and $\tau>0$. Other classes are more easily described by referring to the impulse frequency (i.f.) $\frac{n_{(s, s+t]}^{\gamma}}{t}$ instead of the dwell time. Given $\rho \geq 0$ we consider the class of sequences having i.f. eventually uniformly upper bounded by $\rho$ :

$$
\overline{\mathcal{S}}(\rho)=\left\{\gamma \in \Gamma: \forall \varepsilon>0, \exists T=T(\gamma, \varepsilon)>0 \text { s.t. } \frac{n_{(s, s+t]}^{\gamma}}{t} \leq \rho+\varepsilon, \forall t \geq T, \forall s \geq 0\right\} .
$$

We will later show (Lemma 3.6) that the class $\overline{\mathcal{S}}(\rho)$ is sufficiently broad for our purposes. Given a sequence $\gamma \in \overline{\mathcal{S}}(\rho)$ it straightforwardly follows that $\lim _{\sup _{t \rightarrow \infty}} \frac{n_{(s, s+t]}^{\gamma}}{t}=L_{s} \leq \rho$ for all $s \geq 0$. Moreover, $L_{s}=L_{0}$ for all $s$. In fact, for $s \geq 0$ and $t>0$ we have that

$$
\frac{n_{(s, s+t]}^{\gamma}}{t}=\frac{n_{(0, s+t]}^{\gamma}}{s+t} \cdot \frac{s+t}{t}-\frac{n_{(0, s]}^{\gamma}}{t}
$$

and then taking limsup as $t$ goes to $\infty$ it follows that

$$
L_{s}=\limsup _{t \rightarrow \infty} \frac{n_{(s, s+t]}^{\gamma}}{t}=\limsup _{t \rightarrow \infty} \frac{n_{(0, s+t]}^{\gamma}}{s+t}=L_{0} .
$$

However, the condition $\lim \sup _{t \rightarrow \infty} \frac{n_{(0, t]}^{\gamma}}{t} \leq \rho$ is not sufficient for ensuring that $\gamma \in \overline{\mathcal{S}}(\rho)$, as Example 3.2 shows.

Example 3.2. Consider $\gamma \in \Gamma$ formed by the concatenation of an infinite number of finite sequences $\left\{\tau_{k}^{1}\right\}_{k=0}^{2},\left\{\tau_{k}^{2}\right\}_{k=0}^{2},\left\{\tau_{k}^{3}\right\}_{k=0}^{5}, \ldots,\left\{\tau_{k}^{\ell}\right\}_{k=0}^{p_{\ell}-1}, \ldots$ The finite sequences $\left\{\tau_{k}^{\ell}\right\}$ are defined as follows:

$$
\begin{array}{rlrl}
\tau_{k}^{1} & :=1+\frac{k}{2} & k & =0,1,2 ; \\
\tau_{k}^{\ell}:=2^{\ell}-1+\frac{k}{p_{\ell}-1} & k=0, \ldots, p_{\ell}-1, \quad \ell=2,3, \ldots ; \\
p_{\ell}=3 \cdot 2^{\ell-2} . & &
\end{array}
$$

For each $\ell \geq 2$, the finite sequence $\left\{\tau_{k}^{\ell}\right\}_{k=0}^{p_{\ell}-1}$ is strictly increasing and contains $p_{\ell}=3 \cdot 2^{\ell-2}$ equally spaced elements within an interval of length 1 ; this follows by evaluating $\tau_{0}^{\ell}=2^{\ell}-1, \tau_{p_{\ell}-1}^{\ell}=2^{\ell}$ and $\tau_{k+1}^{\ell}-\tau_{k}^{\ell}=$ $\frac{1}{p_{\ell}-1}=: \Delta_{\ell}$. Note also that $3=\tau_{0}^{2}>\tau_{2}^{1}=2$ and that for $\ell \geq 2$, we have $\tau_{0}^{\ell+1}=2^{\ell+1}-1>\tau_{p_{\ell}-1}^{\ell}=2^{\ell}$. Therefore, the concatenation $\gamma=\left\{\left\{\tau_{k}^{1}\right\},\left\{\tau_{k}^{2}\right\}, \ldots\right\}$ yields a strictly increasing sequence with no finite limit points and hence $\gamma \in \Gamma$. Let $t \geq 2$ and $\ell=\left\lceil\log _{2}(t)\right\rceil$, then

$$
\frac{n_{(0, t]}^{\gamma}}{t} \leq \frac{n_{\left(0,2^{\ell}\right]}^{\gamma}}{2^{\ell}-1}=\frac{3 \cdot 2^{\ell-1}}{2^{\ell}-1}
$$

and therefore $\lim \sup _{t \rightarrow \infty} \frac{n_{(0, t]}^{\gamma}}{t} \leq \frac{3}{2}$. Since given any finite length $T>0$ and arbitrarily large number $N$, we can always find $s \geq 0$ such that $n_{(s, s+T]}^{\gamma} \geq N$, it follows that $\gamma \notin \overline{\mathcal{S}}(\rho)$ for any $\rho>0$. 
A sufficient condition for $\gamma \in \overline{\mathcal{S}}(\rho)$ is that

$$
\limsup _{t \rightarrow \infty} \frac{n_{(s, s+t]}^{\gamma}}{t}=L
$$

uniformly w.r.t. (u.w.r.t.) $s \geq 0$ with $L \leq \rho$ (see Lemma 3.6). We thus define

$$
\hat{\mathcal{S}}(L):=\left\{\gamma \in \Gamma: \limsup _{t \rightarrow \infty} \frac{n_{(s, s+t]}^{\gamma}}{t}=L \text {, u.w.r.t. } s \geq 0\right\} .
$$

\subsection{Classes involving lower bounds}

We can have some analogous definitions involving lower bounds, such as reverse average dwell time with reverse chatter bound $\mathrm{n} \in \mathbb{N}$ and reverse average dwell time $\tau>0$ :

$$
\mathcal{S}_{\mathrm{RAD}}(\mathrm{n}, \tau)=\left\{\gamma \in \Gamma: n_{(s, t]}^{\gamma} \geq-\mathrm{n}+\frac{t-s}{\tau}, \forall 0 \leq s \leq t\right\},
$$

and the class of sequences having i.f. eventually uniformly lower bounded by $\rho$ :

$$
\underline{\mathcal{S}}(\rho)=\left\{\gamma \in \Gamma: \forall \varepsilon>0, \exists T=T(\gamma, \varepsilon)>0 \text { s.t. } \frac{n_{(s, s+t]}^{\gamma}}{t} \geq \rho-\varepsilon, \forall t \geq T, \forall s \geq 0\right\} .
$$

In this case, a sufficient condition for $\gamma \in \underline{\mathcal{S}}(\rho)$ is that

$$
\liminf _{t \rightarrow \infty} \frac{n_{(s, s+t]}^{\gamma}}{t}=L
$$

u.w.r.t. $s \geq 0$ with $L \geq \rho$ (see Lemma 3.6) and we define

$$
\check{\mathcal{S}}(L):=\left\{\gamma \in \Gamma: \liminf _{t \rightarrow \infty} \frac{n_{(s, s+t]}^{\gamma}}{t}=L, \text { u.w.r.t. } s \geq 0\right\} .
$$

\subsection{Classes involving upper and lower bounds}

For comparison of our results, we define the class of impulse-time sequences with fixed dwell times:

$$
\mathcal{S}_{\mathrm{F}}\left(\theta_{1}, \theta_{2}\right)=\left\{\gamma=\left\{\tau_{k}\right\}_{k=1}^{\infty} \in \Gamma: \theta_{1} \leq \tau_{k+1}-\tau_{k} \leq \theta_{2}, \forall k \in \mathbb{N}\right\} .
$$

and that of sequences having i.f. with a uniform limit $\rho$ :

$$
\mathcal{S}_{\mathrm{LIM}}^{u}(\rho)=\left\{\gamma \in \Gamma: \lim _{t \rightarrow \infty} \frac{n_{(s, s+t]}^{\gamma}}{t}=\rho \text { u.w.r.t. } s \geq 0\right\} .
$$

It is clear that $\mathcal{S}_{\text {LIM }}^{u}(\rho) \subset \overline{\mathcal{S}}(\rho) \cap \underline{\mathcal{S}}(\rho)$ and hence is a much smaller class than either $\overline{\mathcal{S}}(\rho)$ or $\underline{\mathcal{S}}(\rho)$.

\subsection{Uniformity and relationships}

The word "uniformly" in the definitions of $\overline{\mathcal{S}}(\rho)$ and $\underline{\mathcal{S}}(\rho)$ refers to uniformity with respect to initial time. In the definition of UIB, however, "uniformly" refers to uniformity with respect to both initial time and every impulse-time sequence in the set. We are also interested in sets of impulse-time sequences where the bounds imposed by $\overline{\mathcal{S}}(\rho)$ or $\underline{\mathcal{S}}(\rho)$ hold uniformly over every sequence in the set. We therefore employ the following definition. 
Definition 3.3. We say that a set of impulse-time sequences $\mathcal{S} \subset \Gamma$ is a uniform subset of $\overline{\mathcal{S}}(\rho)$ (resp. $\underline{\mathcal{S}}(\rho)$ ), and write $\mathcal{S} 匹 \overline{\mathcal{S}}(\rho)$ (resp. $\mathcal{S} 匹 \underline{\mathcal{S}}(\rho))$, if $\mathcal{S} \subset \overline{\mathcal{S}}(\rho)$ (resp. $\mathcal{S} \subset \underline{\mathcal{S}}(\rho)$ ) and $T$ in (6) (resp. (7)) can be selected independently of $\gamma \in \mathcal{S}$.

Remark 3.4. Every $\mathcal{S} \subset \overline{\mathcal{S}}(\rho)(\mathcal{S} \subset \underline{\mathcal{S}}(\rho))$ containing a finite number of sequences satisfies $\mathcal{S} 匹 \overline{\mathcal{S}}(\rho)$ $(\mathcal{S} 匹 \underline{\mathcal{S}}(\rho))$ because the required $T$ can be taken as $\max _{\gamma \in \mathcal{S}} T(\gamma, \varepsilon)$.

In Lemma 3.6, we prove that the classes $\overline{\mathcal{S}}(\rho)$ and $\underline{\mathcal{S}}(\rho)$ are very broad, i.e., many known classes of impulse-time sequences are (uniform) subsets of the classes with eventually uniformly upper/lower bounded i.f. We require the following definition.

Definition 3.5. Given $\Delta \geq 0$, we say that $\gamma^{*}=\left\{\tau_{k}^{*}\right\}_{k=1}^{N} \in \Gamma(N \in \mathbb{N}$ or $N=\infty)$ is a $\Delta$-perturbation of $\gamma=\left\{\tau_{k}\right\}_{k=1}^{N} \in \Gamma$ if $\tau_{k}^{*} \in\left[\tau_{k}-\Delta, \tau_{k}+\Delta\right]$ for all $k$.

Lemma 3.6. The following relationships hold.

i) $\mathcal{S}_{\mathrm{AD}}(\mathrm{n}, \tau) 匹 \overline{\mathcal{S}}\left(\frac{1}{\tau}\right)$ and $\mathcal{S}_{\mathrm{RAD}}(\mathrm{n}, \tau) 匹 \underline{\mathcal{S}}\left(\frac{1}{\tau}\right)$.

ii) If $\mathcal{S} 匹 \overline{\mathcal{S}}(\rho)(\mathcal{S} \llbracket \underline{\mathcal{S}}(\rho))$, then for each $0<\tau<\frac{1}{\rho}\left(\tau>\frac{1}{\rho}\right)$ there exists $\mathrm{n}$ such that $\mathcal{S} \subset \mathcal{S}_{\mathrm{AD}}(\mathrm{n}, \tau)$ $\left(\mathcal{S} \subset \mathcal{S}_{\mathrm{RAD}}(\mathrm{n}, \tau)\right)$.

iii) $\hat{\mathcal{S}}(L) \subset \overline{\mathcal{S}}(\rho)(\check{\mathcal{S}}(L) \subset \underline{\mathcal{S}}(\rho))$ for all $L \leq \rho(L \geq \rho)$.

iv) $\mathcal{S}_{\mathrm{F}}\left(\theta_{1}, \theta_{2}\right) \lll \overline{\mathcal{S}}\left(\frac{1}{\theta_{1}}\right)$ and $\mathcal{S}_{\mathrm{F}}\left(\theta_{1}, \theta_{2}\right) 匹 \underline{\mathcal{S}}\left(\frac{1}{\theta_{2}}\right)$.

v) $\overline{\mathcal{S}}(\rho)$ and $\underline{\mathcal{S}}(\rho)$ are persistent under perturbations:

a) if $\gamma^{*}$ is a $\Delta$-perturbation of $\gamma \in \overline{\mathcal{S}}(\rho)(\gamma \in \underline{\mathcal{S}}(\rho))$, then $\gamma^{*} \in \overline{\mathcal{S}}(\rho)\left(\gamma^{*} \in \underline{\mathcal{S}}(\rho)\right)$;

b) if $\mathcal{S} 匹 \overline{\mathcal{S}}(\rho)(\mathcal{S} 匹 \underline{\mathcal{S}}(\rho))$, then $\mathcal{S}_{\Delta}$, the set of all the $\Delta$-perturbations of sequences in $\mathcal{S}$, satisfies $\mathcal{S}_{\Delta} 匹 \overline{\mathcal{S}}(\rho)\left(\mathcal{S}_{\Delta} 匹 \underline{\mathcal{S}}(\rho)\right)$.

Proof. (i) Consider any $\gamma \in \mathcal{S}_{\mathrm{AD}}(\mathrm{n}, \tau)$. Then,

$$
n_{(s, t]}^{\gamma} \leq \mathrm{n}+\frac{t-s}{\tau} \text { for all } 0 \leq s<t .
$$

Therefore,

$$
\frac{n_{(s, s+t]}^{\gamma}}{t} \leq \frac{\mathrm{n}}{t}+\frac{1}{\tau} \quad \forall s \geq 0, \forall t>0
$$

Given $\varepsilon>0$, let $T=T(\varepsilon):=\frac{\mathrm{n}}{\varepsilon}$. Then, $\frac{n_{(s, s+t)}^{\gamma}}{t} \leq \frac{1}{\tau}+\varepsilon$ holds for all $s \geq 0$ and $t \geq T$. Hence $\gamma \in \overline{\mathcal{S}}\left(\frac{1}{\tau}\right)$. Since $T$ does not depend on the specific $\gamma$ considered, we conclude that $\mathcal{S}_{\mathrm{AD}}(\mathrm{n}, \tau) 匹 \overline{\mathcal{S}}\left(\frac{1}{\tau}\right)$. The inclusion $\mathcal{S}_{\mathrm{RAD}}(\mathrm{n}, \tau) 匹 \underline{\mathcal{S}}\left(\frac{1}{\tau}\right)$ can be proved analogously.

iii) By assumption, for every $\varepsilon>0$ there exists $T=T(\varepsilon)>0$ such that $\frac{n_{(s, s+t]}^{\gamma}}{t} \leq \rho+\varepsilon$ holds for all $t \geq T$, $s \geq 0$ and $\gamma \in \mathcal{S}$. For $0 \leq t<T$, it holds that

$$
n_{(s, s+t]}^{\gamma} \leq n_{(s, s+T]}^{\gamma} \leq(\rho+\varepsilon) T \quad \forall s \geq 0, \forall \gamma \in \mathcal{S} .
$$

Combining the bounds for small and large $t$ yields

$$
n_{(s, s+t]}^{\gamma} \leq(\rho+\varepsilon) T+(\rho+\varepsilon) t \quad \forall s, t \geq 0, \forall \gamma \in \mathcal{S} .
$$

Let $0<\tau<\frac{1}{\rho}$, set $\varepsilon=1 / \tau-\rho>0$ and take the corresponding $T=T(\varepsilon)$. Substituting these quantities into (9), it follows that $n_{(s, s+t]}^{\gamma} \leq T / \tau+t / \tau$. Taking $\mathrm{n}=\lceil T / \tau\rceil$, then $\mathcal{S} \subset \mathcal{S}_{\mathrm{AD}}(\mathrm{n}, \tau)$. The other case can be proved analogously. 
iiii) Let $\gamma \in \hat{\mathcal{S}}(L)$ with $L \leq \rho$ and $\varepsilon>0$. By definition of $\hat{\mathcal{S}}(L)$, there exists $T=T(\gamma, \varepsilon)>0$ such that

$$
L-\varepsilon \leq \sup _{\tau \geq t} \frac{n_{(s, s+\tau]}^{\gamma}}{\tau} \leq L+\varepsilon \quad \forall s \geq 0, \forall t \geq T
$$

Then $\frac{n_{(s, s+t)}^{\gamma}}{t} \leq \rho+\varepsilon$ for all $t \geq T$ and hence $\gamma \in \overline{\mathcal{S}}(\rho)$. The other inclusion can be proved analogously.

iv) This follows from item (ii) and the facts that $\mathcal{S}_{\mathrm{F}}\left(\theta_{1}, \theta_{2}\right) \subset \mathcal{S}_{\mathrm{AD}}\left(1, \theta_{1}\right)$ and $\mathcal{S}_{\mathrm{F}}\left(\theta_{1}, \theta_{2}\right) \subset \mathcal{S}_{\mathrm{RAD}}\left(1, \theta_{2}\right)$.

v)a Pick any $\gamma \in \overline{\mathcal{S}}(\rho)$ and let $\gamma^{*}$ be a $\Delta$-perturbation of $\gamma$. Let $\varepsilon_{1}>0$ and define $\varepsilon=\varepsilon_{1} / 2$. Since $\gamma \in \mathcal{S}(\rho)$ then there exists $T=T(\gamma, \varepsilon)>0$ such that

$$
\frac{n_{(s, s+t]}^{\gamma}}{t} \leq \rho+\varepsilon \quad \forall t \geq T, \forall s \geq 0 .
$$

Let us pick some $t^{*}>\max \{\Delta, T\}$. Then,

$$
\frac{n_{\left(s, s+t^{*}\right]}^{\gamma}}{t^{*}}=\frac{n_{(s, s+\Delta]}^{\gamma}+n_{\left(s+\Delta, s+t^{*}\right]}^{\gamma}}{t^{*}} \leq \rho+\varepsilon \forall s \geq 0
$$

and, hence,

$$
n_{(s, s+\Delta]}^{\gamma} \leq(\rho+\varepsilon) t^{*}-n_{\left(s+\Delta, s+t^{*}\right]}^{\gamma} \leq(\rho+\varepsilon) t^{*} \forall s \geq 0 .
$$

For any $s, t \geq 0$ denote by $n_{(s, s+t]}^{+}=n_{(s, s+t]}^{\gamma^{*}}-n_{(s, s+t]}^{\gamma}$. By definition of $\Delta$-perturbation, it follows that

$$
\left|n_{(s, s+t]}^{+}\right| \leq 2 \sup _{r \geq 0} n_{(r, r+\Delta]}^{\gamma} \quad \forall s, t \geq 0 .
$$

Then, adding $\frac{n_{(s, s+t)}^{+}}{t}$ to the left and right-hand sides of (10), we get

$$
\frac{n_{(s, s+t]}^{\gamma^{*}}}{t} \leq \rho+\varepsilon+\frac{n_{(s, s+t]}^{+}}{t} \leq \rho+\varepsilon+\frac{2 \sup _{r \geq 0} n_{(r, r+\Delta]}^{\gamma}}{t} \leq \rho+\varepsilon+\frac{2(\rho+\varepsilon) t^{*}}{t} \quad \forall t \geq T, \forall s \geq 0 .
$$

Let $T_{1}=\frac{2(\rho+\varepsilon) t^{*}}{\varepsilon_{1}-\varepsilon}=\frac{4(\rho+\varepsilon) t^{*}}{\varepsilon_{1}}>0$. Then, $\frac{n_{(s, s+t]}^{\gamma^{*}}}{t} \leq \rho+\varepsilon_{1}$ for all $t \geq T_{1}$ and $s \geq 0$; hence, $\gamma^{*} \in \overline{\mathcal{S}}(\rho)$.

v)b This follows from the proof of v)a by noting that if $\gamma \in \mathcal{S} 匹 \overline{\mathcal{S}}(\rho)$, then $T$ and hence $t^{*}$ can be chosen independently of $\gamma$. This causes $T_{1}$ to become independent of $\gamma^{*} \in \mathcal{S}_{\Delta}(\rho)$. As a consequence, $\mathcal{S}_{\Delta} 匹 \overline{\mathcal{S}}(\rho)$.

The implications involving $\underline{\mathcal{S}}(\rho)$ can be proved analogously.

By means of Lemma 3.6 , we may see that $\overline{\mathcal{S}}(\rho)$ and $\underline{\mathcal{S}}(\rho)$ incorporate impulse-time sequences with

- (reverse) average dwell-time (Hespanha et al., 2005, 2008), Lemma 3.6illii);

- i.f. eventually uniformly (upper/lower) convergent (Dashkovskiv and Feketa, 2017; Feketa and Baicinca, 2019a), Lemma 3.6liii);

- fixed dwell-time (Samoilenko and Perestyuk, 1987; Dashkovskiy and Mironchenko, 2013), Lemma 3.(1lv);

- non-fixed impulse-time moments within predefined time-windows (Tan et al., 2015; Feng et al., 2017; Feketa and Baicinca, 2019b), Lemma (3.6( $)$.

In order to gain more insight into the breadth of the classes $\overline{\mathcal{S}}(\rho)$ and $\underline{\mathcal{S}}(\rho)$, and the facts given by Lemma 3.(1) iii), we remark that for every $\tau>0$, the sets $\overline{\mathcal{S}}\left(\frac{1}{\tau}\right)$ and $\underline{\mathcal{S}}\left(\frac{1}{\tau}\right)$ contain sequences which do not belong to, respectively, $\mathcal{S}_{\mathrm{AD}}(\mathrm{n}, \tau)$ and $\mathcal{S}_{\mathrm{RAD}}(\mathrm{n}, \tau)$ for any $\mathrm{n} \in \mathbb{N}$. Example 3.7 illustrates this fact for the case $\tau=1$. Examples for arbitrary values of $\tau$ can be obtained by simple modifications. 
Example 3.7. Consider $\gamma=\left\{\tau_{k}\right\}_{k=1}^{\infty}$ defined by

$$
\tau_{1}=1 \quad \text { and } \quad \tau_{k}=k-\sum_{\ell=2}^{k} \frac{1}{\ell} \text { for } k \geq 2,
$$

so that $\Delta_{k}:=\tau_{k}-\tau_{k-1}=1-1 / k$ for all $k \geq 2$. Let $\varepsilon>0$ and define $\tau^{\prime}:=\frac{1}{1+\varepsilon / 2}$. Note that $0<\tau^{\prime}<1$. Since $\Delta_{k} \nearrow 1$, then there exists $k_{0} \geq 2$ so that $1>\Delta_{k} \geq \tau^{\prime}$ for all $k \geq k_{0}$. Set $n_{0}:=n_{\left(0, k_{0}\right]}^{\gamma}$ and $T=T(\varepsilon):=2 \frac{n_{0}+1}{\varepsilon}$. For every $s \geq k_{0}$, we have that $\tau_{k} \geq s$ implies that $k \geq k_{0}$. In consequence, for all $s \geq k_{0}$ and $t>0$

$$
n_{(s, s+t]}^{\gamma} \leq 1+\frac{t}{\tau^{\prime}}, \quad \text { and then } \quad \frac{n_{(s, s+t]}^{\gamma}}{t} \leq \frac{1}{t}+\frac{1}{\tau^{\prime}}
$$

For $0 \leq s<k_{0}$ and $t>0$,

$$
\frac{n_{(s, s+t]}^{\gamma}}{t} \leq \frac{n_{\left(0, k_{0}+t\right]}^{\gamma}}{t} \leq \frac{n_{0}+n_{\left(k_{0}, k_{0}+t\right]}^{\gamma}}{t} \leq \frac{n_{0}+1}{t}+\frac{1}{\tau^{\prime}}
$$

Therefore, for all $s \geq 0$ and $t>0$ it follows that

$$
\frac{n_{(s, s+t]}^{\gamma}}{t} \leq \frac{n_{0}+1}{t}+\frac{1}{\tau^{\prime}}
$$

Recalling the definitions of $\tau^{\prime}$ and $T$, we arrive to

$$
\frac{n_{(s, s+t]}^{\gamma}}{t} \leq 1+\varepsilon \quad \forall s \geq 0, t \geq T .
$$

We have thus shown that $\gamma \in \overline{\mathcal{S}}(1)$. Next, we prove that for every $\mathrm{n} \in \mathbb{N}, \gamma \notin \mathcal{S}_{\mathrm{AD}}(\mathrm{n}, 1)$. Suppose for a contradiction that $\gamma \in \mathcal{S}_{\mathrm{AD}}\left(\mathrm{n}^{*}, 1\right)$ for some $\mathrm{n}^{*} \in \mathbb{N}$. By definition of $\mathcal{S}_{\mathrm{AD}}$ then

$$
n_{(0, t]}^{\gamma} \leq \mathrm{n}^{*}+t \text { for all } t>0
$$

Let $k \in \mathbb{N}$ with $k \geq 2$. Since $\tau_{k}=k-\sum_{\ell=2}^{k} \frac{1}{\ell}$ then

$$
n_{(0, k]}^{\gamma}=k+n_{\left(\tau_{k}, k\right]}^{\gamma} \geq k+\left\lfloor\sum_{\ell=2}^{k} \frac{1}{\ell}\right\rfloor .
$$

Consider $k$ sufficiently large so that $\sum_{\ell=2}^{k} \frac{1}{\ell}>\mathrm{n}^{*}+1$ and let $t=k$. Then, (11) gives $n_{(0, k]}^{\gamma} \leq k+\mathrm{n}^{*}$ but (12) gives $n_{(0, k]}^{\gamma} \geq k+\mathrm{n}^{*}+1$. This is clearly a contradiction.

Arguments similar to those used in Example 3.7 show that the sequence $\gamma=\left\{\tau_{k}\right\}_{k=1}^{\infty}$ defined recursively by $\tau_{1}=1$ and $\tau_{k}=k+\sum_{\ell=2}^{k}(1 / \ell)$ for $k \geq 2$ satisfies $\gamma \in \underline{\mathcal{S}}(1)$ and $\gamma \notin \mathcal{S}_{\mathrm{RAD}}(\mathrm{n}, 1)$ for any $\mathrm{n} \in \mathbb{N}$.

The following example illustrates a class $\mathcal{S} \subset \Gamma$ that is a subset of $\overline{\mathcal{S}}(1)$ but not a uniform subset.

Example 3.8. For each $\mathrm{n} \in \mathbb{N}$ with $\mathrm{n} \geq 2$, consider a sequence $\gamma_{\mathrm{n}} \in \Gamma$ constructed by concatenating an infinite number of finite sequences $\left\{\tau_{\mathrm{n}, k}^{\ell}\right\}_{k=0}^{\mathrm{n}-1}$, for $\ell=1,2, \ldots$, whose elements are defined as follows

$$
\tau_{\mathrm{n}, k}^{\ell}=1+(\ell-1) \mathrm{n}+\frac{k}{\ell-1+\mathrm{n}} .
$$

For $0 \leq k \leq \mathrm{n}-2$, we have

$$
\tau_{\mathrm{n}, k+1}^{\ell}-\tau_{\mathrm{n}, k}^{\ell}=\frac{1}{\ell-1+\mathrm{n}}
$$

so that the spacing between consecutive elements in the finite sequence $\left\{\tau_{\mathrm{n}, k}^{\ell}\right\}_{k=0}^{\mathrm{n}-1}$ becomes smaller and smaller as $\ell \rightarrow \infty$ because $\lim _{\ell \rightarrow \infty} \tau_{\mathrm{n}, k+1}^{\ell}-\tau_{\mathrm{n}, k}^{\ell}=0$. It can be shown that $\gamma_{\mathrm{n}} \in \mathcal{S}_{\mathrm{AD}}(\mathrm{n}, 1)$. From Lemma 3.(11i), then $\gamma_{\mathrm{n}} \in \overline{\mathcal{S}}(1)$ for every $\mathrm{n}$. Therefore, the class $\mathcal{S}:=\left\{\gamma_{\mathrm{n}}: \mathrm{n} \in \mathbb{N}, \mathrm{n} \geq 2\right\}$ satisfies $\mathcal{S} \subset \overline{\mathcal{S}}(1)$. However, $\mathcal{S}$ is not a uniform subset of $\overline{\mathcal{S}}(1)$ because, from the proof of Lemma 3.(6ili) then the required $T$ in (6) cannot be taken independent of $\mathrm{n}$ and hence cannot be independent of $\gamma_{\mathrm{n}}$. 


\section{ISS under eventually uniformly bounded i.f.}

In this section, we provide sufficient conditions for the weak and strong $\left(h^{o}, h\right)$-ISS over classes of impulsetime sequences having eventually uniformly bounded impulse frequency. Our main results are stated in Section 4.1. In Section 4.2, we explain our proof technique, based on the analysis of a comparison system. The remaining technical results required for the full proof are given in Section 4.3 .

\subsection{Main results}

We say that a locally Lipschitz function $V: \mathbb{R}_{\geq 0} \times \mathbb{R}^{n} \rightarrow \mathbb{R}$ is a $\left(h^{o}, h\right)$-ISS Lyapunov function candidate for system (1) if

a) there exist $\phi_{1}, \phi_{2} \in \mathcal{K}_{\infty}$ so that for all $t \geq 0$ and $\xi \in \mathbb{R}^{n}$,

$$
\phi_{1}(h(t, \xi)) \leq V(t, \xi) \leq \phi_{2}\left(h^{o}(t, \xi)\right) ;
$$

b) there exist $\chi, \pi \in \mathcal{K}_{\infty}$, a locally integrable function $p: \mathbb{R}_{>0} \rightarrow \mathbb{R}_{\geq 0}$, a continuous function $\varphi: \mathbb{R}_{>0} \rightarrow \mathbb{R}$ and $\psi \in \mathcal{P}$ such that for all $t \geq 0, \xi \in \mathbb{R}^{n}$ and $\mu \in \mathbb{R}^{m}$,

i) $D_{f}^{+} V(t, \xi, \mu) \leq-p(t) \varphi(V(t, \xi))$ if $V(t, \xi) \geq \chi(|\mu|)$;

ii) $V(t, \xi+g(t, \xi, \mu)) \leq \psi(V(t, \xi))$ if $V(t, \xi) \geq \chi(|\mu|)$;

iii) $V(t, \xi+g(t, \xi, \mu)) \leq \pi(|\mu|)$ if $V(t, \xi) \leq \chi(|\mu|)$.

Here, $D_{f}^{+} V(t, \xi, \mu)$ denotes the upper-right Dini derivative of $V$ along $f$ at $(t, \xi, \mu) \in \mathbb{R}_{\geq 0} \times \mathbb{R}^{n} \times \mathbb{R}^{m}$, i.e.

$$
D_{f}^{+} V(t, \xi, \mu):=\limsup _{h \rightarrow 0^{+}} \frac{V(t+h, \xi+h f(t, \xi, \mu))-V(t, \xi)}{h} .
$$

Theorem 4.1. Let $V$ be $a\left(h^{\circ}, h\right)$-ISS Lyapunov function candidate for system (1) with $\psi, \varphi \in \mathcal{P}$. Suppose that there exists $\theta>0$ such that

$$
M:=\sup _{a>0} \int_{a}^{\psi(a)} \frac{d s}{\varphi(s)}<\inf _{t \geq 0} \int_{t}^{t+\theta} p(s) d s=: N
$$

Then, the following hold.

a) If $M>0$, then (1) is strongly $\left(h^{o}, h\right)$-ISS over any $\mathcal{S} \subset \Gamma$ such that $\mathcal{S} 匹 \overline{\mathcal{S}}\left(\frac{1}{\theta}\right)$.

b) If $M=0$, then (1) is weakly $\left(h^{o}, h\right)$-ISS over $\mathcal{S}=\Gamma$ and strongly $\left(h^{\circ}, h\right)$-ISS over any UIB family $\mathcal{S} \subset \Gamma$.

c) If $M<0<N$, then (1) is strongly $\left(h^{\circ}, h\right)$-ISS over $\mathcal{S}=\Gamma$.

Theorem 4.1 gives sufficient conditions for $\left(h^{o}, h\right)$-ISS when the continuous part of the dynamics cannot be destabilizing for large values of the state. The case $M<0$ corresponds to stabilizing impulses and hence the ensuing stability is very strong. The most interesting case is $M>0$, corresponding to destabilizing impulses. Inversely to Theorem 4.1, Theorem 4.2 addresses the case when the continuous part cannot be stabilizing.

Theorem 4.2. Let $V$ be $a\left(h^{o}, h\right)$-ISS Lyapunov function candidate for system (1) with $\psi,-\varphi \in \mathcal{P}$. Let $\mathcal{S} \subset \Gamma$ satisfy $\mathcal{S} 匹 \underline{\mathcal{S}}\left(\frac{1}{\theta}\right)$ with $\theta>0$ such that

$$
\inf _{a>0} \int_{\psi(a)}^{a} \frac{d s}{-\varphi(s)}>\sup _{t \geq 0} \int_{t}^{t+\theta} p(s) d s .
$$

Suppose that, in addition,

$$
\int_{1}^{\infty} \frac{d s}{-\varphi(s)}=\infty .
$$

Then (1) is strongly $\left(h^{\circ}, h\right)$-ISS over $\mathcal{S}$. 
Theorems 4.1 and 4.2 provide a manifold extension of the results for the stability analysis of impulsive systems available in the literature. More precisely, these theorems extend the results in Mancilla-Aguilar and Haimovich (2019); Feketa and Bajcinca (2019b)a) to provide two-measure uniform ISS results for time-varying nonlinear impulsive systems over sequences having eventually uniformly bounded impulse frequency.

The results in Mancilla-Aguilar and Haimovich (2019, Sections 4, 5), in turn, provide sufficient conditions for two-measure ISS that constituted a substantial extension of previously available results. The reader may refer to Mancilla-Aguilar and Haimovich (2019) for specific explanations on how existing results were extended by the latter. In particular, Theorem 4.4 in Mancilla-Aguilar and Haimovich (2019) provides sufficient conditions for the $\left(h^{\circ}, h\right)$-ISS of a system of the form (1) based on a Lyapunov function candidate of the type considered here but where ISS holds uniformly only over sequences with fixed dwell times. The extension from fixed dwell-time sequences to sequences having eventually uniformly bounded i.f. is substantial, as shown by Lemma 3.6. In addition, excepting for the class of impulse-time sequences, the sufficient conditions of Theorems 4.1 and 4.2 coincide with those of Theorem 4.4 in Mancilla-Aguilar and Haimovich (2019), and hence in this case the current results strengthen the corresponding conclusions.

The results in Feketa and Bajcinca (2019b) provide nonuniform GAS results over sequences having nonfixed impulse times over predefined time windows and those in Feketa and Bajcinca (2019a) provide nonuniform ISS results over sequences having a uniform limit, i.e. sequences $\gamma \in \mathcal{S}_{\mathrm{LIM}}^{u}(L)$ for some $L>0$. The extension with respect to the latter results regards uniformity with respect to initial time and the (broader) class of impulse-time sequences considered, two-measure ISS, and the consideration of Lyapunov function candidates with possibly time-varying rates.

\subsection{Proof technique}

If $V$ is a Lyapunov function candidate for system (11), we consider the following one-dimensional differential / difference inclusion system, which we henceforth call comparison system:

$$
\begin{array}{rlrl}
\dot{z}(t) & \in(-\infty,-p(t) \varphi(z(t))], & & t \notin \gamma, \\
z(t) & \in\left[0, \psi\left(z\left(t^{-}\right)\right)\right], & t \in \gamma .
\end{array}
$$

We say that a function $z: I_{z} \rightarrow \mathbb{R}_{\geq 0}$, with $I_{z}=\left[t_{0}, T_{z}\right)$ is a solution of (17) corresponding to $\gamma=\left\{\tau_{k}\right\} \in \Gamma$, initial time $t_{0} \geq 0$ and initial condition $z_{0} \geq 0$ if i) $z\left(t_{0}\right)=z_{0}$, ii) for every nonempty interval $J_{k}=$ $\left[\tau_{k}, \tau_{k+1}\right) \cap I_{z}, z$ is locally absolutely continuous on $J_{k}$ and $\dot{z}(t) \leq-p(t) \varphi(z(t))$ for almost all $t \in J_{k}$, and iii) for every $\tau_{k} \in \gamma \cap\left(t_{0}, T_{z}\right)$, it happens that $z\left(\tau_{k}^{-}\right)$exists and $0 \leq z\left(\tau_{k}\right) \leq \psi\left(z\left(\tau_{k}^{-}\right)\right)$. A solution $z$ of (17) is maximally defined if it does not have a proper extension; it is forward complete if $T_{z}=\infty$. We will employ $\mathcal{C}\left(t_{0}, z_{0}, \gamma\right)$ to denote the set of maximally defined solutions $z$ of (17) corresponding to $\gamma \in \Gamma$, initial time $t_{0}$ and initial condition $z_{0}$. Note that, by definition, every solution of the comparison system is nonnegative.

We say that the comparison system (17) is weakly or strongly GUAS (uniformly) over $\mathcal{S} \subset \Gamma$ if there exists a function $\beta \in \mathcal{K} \mathcal{L}$ such that every $z \in \mathcal{C}\left(t_{0}, z_{0}, \gamma\right)$ with $\gamma \in \mathcal{S}, t_{0} \geq 0$ and $z_{0} \geq 0$ satisfies, respectively

$$
\begin{aligned}
\text { (weak) } & z(t) \leq \beta\left(z_{0}, t-t_{0}\right) & \forall t \in I_{z}, \\
\text { (strong) } & z(t) \leq \beta\left(z_{0}, t-t_{0}+n_{\left(t_{0}, t\right]}^{\gamma}\right) & \forall t \in I_{z} .
\end{aligned}
$$

Section 3 of Mancilla-Aguilar and Haimovich (2019) gives the basis method for ensuring that system (1) is weakly or strongly $\left(h^{o}, h\right)$-ISS over $\mathcal{S}$ by analyzing the comparison system (17) given by means of an $\left(h^{o}, h\right)$ ISS Lyapunov function candidate. Specifically, Theorem 3.1 in Mancilla-Aguilar and Haimovich (2019) states that system (11) is weakly or strongly $\left(h^{o}, h\right)$-ISS over some arbitrary class $\mathcal{S}$ provided the corresponding comparison system is, respectively, weakly or strongly GUAS uniformly over $\mathcal{S}$. In the current paper, our main proof technique consists in establishing the required type of GUAS for the comparison system and applying Theorem 3.1 in Mancilla-Aguilar and Haimovich (2019) to ensure the required type of $\left(h^{o}, h\right)$-ISS. Propositions 4.3 and 4.4 below provide the required GUAS results and hence constitute our main technical contribution. Their proofs are given in Section 4.3 .

Proposition 4.3. Let $\psi, \varphi \in \mathcal{P}$ and $p: \mathbb{R}_{\geq 0} \rightarrow \mathbb{R}_{\geq 0}$ be locally integrable. Let $\theta>0$ be such that (14) holds. Then, the following hold. 
a) If $M>0$, then the comparison system (17) is strongly $G U A S$ uniformly over any $\mathcal{S} 匹 \mathcal{\mathcal { S }}\left(\frac{1}{\theta}\right)$.

b) If $M=0$, then the comparison system (17) is weakly GUAS uniformly over $\mathcal{S}=\Gamma$ and strongly $G U A S$ uniformly over any UIB family $\mathcal{S} \subset \Gamma$.

c) If $M<0<N$, then the comparison system (17) is strongly GUAS uniformly over $\mathcal{S}=\Gamma$.

Proposition 4.4. Let $\psi,-\varphi \in \mathcal{P}, p: \mathbb{R}_{\geq 0} \rightarrow \mathbb{R}_{\geq 0}$ be locally integrable, and $\theta>0$ be such that (15)-(16) are satisfied. Then, (17) is strongly GUAS uniformly over $\mathcal{S}$ for every $\mathcal{S} \subset \Gamma$ such that $\mathcal{S} 匹 \underline{\mathcal{S}}^{u}\left(\frac{1}{\theta}\right)$.

Proof (Theorems 4.1 And 4.2). As previously mentioned, the proofs of Theorems 4.1 and 4.2 follow straightforwardly from Propositions 4.3 and 4.4, respectively, and Theorem 3.1 in Mancilla-Aguilar and Haimovich (2019). Employing the notation and definitions in Mancilla-Aguilar and Haimovich (2019), consider the parametrized family of impulsive systems $\left\{\Sigma_{\gamma}\right\}_{\gamma \in \mathcal{S}}$, where $\Sigma_{\gamma}=\left(\gamma, f_{\gamma}, g_{\gamma}\right)$, with $f_{\gamma}=f$ and $g_{\gamma}=g$ for all $\gamma \in \mathcal{S}$. Here, we employ the family $\mathcal{S}$ of impulse-time sequences as the parameter set and the sequence $\gamma$ as a parameter (see Section II.B in Mancilla-Aguilar and Haimovich, 2019). Also consider the family of functions $\left\{V_{\gamma}\right\}_{\gamma \in \mathcal{S}}$ with $V_{\gamma}=V$ for all $\gamma \in \mathcal{S}$, where $V$ is the $\left(h^{o}, h\right)$-ISS Lyapunov function candidate appearing in the hypotheses of both Theorem 4.1 and 4.2. Then, $\left\{V_{\gamma}\right\}_{\gamma \in \mathcal{S}}$ satisfies Assumption 1 in Mancilla-Aguilar and Haimovich (2019), and the family of comparison systems associated with $\left\{\Sigma_{\gamma}\right\}_{\gamma \in \mathcal{S}}$ and $\left\{V_{\gamma}\right\}_{\gamma \in \mathcal{S}}$ (defined in eq. (7) of Mancilla-Aguilar and Haimovich, 2019) coincides with the comparison system (17). Applying Theorem 3.1 of Mancilla-Aguilar and Haimovich (2019) to the family of systems $\left\{\Sigma_{\gamma}\right\}_{\gamma \in \mathcal{S}}$, then the system (11) is weakly or strongly $\left(h^{o}, h\right)$-ISS over $\mathcal{S}$ when the comparison system (17) is weakly or strongly GUAS over $\mathcal{S}$, respectively. Finally, the weak or strong GUAS over $\mathcal{S}$ of the comparison system is established in Propositions 4.3 and 4.4 in each of the considered cases.

\subsection{Remaining proofs}

It can be shown, following the lines of Proposition 2.5 in Lin et al. (1996), that (17) is weakly GUAS uniformly over $\mathcal{S}$ if and only if the following hold

ci) (Global uniform stability, GUS) there exists $\alpha \in \mathcal{K}$ such that

$$
|z(t)| \leq \alpha\left(\left|z\left(t_{0}\right)\right|\right) \quad \forall t \in I_{z},
$$

for all $t_{0} \geq 0, z_{0} \geq 0, \gamma \in \mathcal{S}$ and $z \in \mathcal{C}\left(t_{0}, z_{0}, \gamma\right)$;

cii) (Uniform attractivity) for all $0<\varepsilon \leq R$ there exists $T=T(\varepsilon, R)>0$ such that for all $t_{0} \geq 0, z_{0} \geq 0$ with $\left|z_{0}\right| \leq R, \gamma \in \mathcal{S}$ and $z \in \mathcal{C}\left(t_{0}, z_{0}, \gamma\right)$ we have that $|z(t)| \leq \varepsilon$ for all $t \in\left[t_{0}+T, \infty\right) \cap I_{z}$. (2019).

The following result can be proved in the same manner as Proposition 2.3 in Mancilla-Aguilar and Haimovich

Lemma 4.5. Let $\mathcal{S} \subset \Gamma$ be UIB. Then (17) is strongly $G U A S$ over $\mathcal{S}$ if and only if it is weakly GUAS over $\mathcal{S}$.

The proof of Proposition 4.3 requires the following results.

Lemma 4.6. Let $\varphi \in \mathcal{P}$ and $p: \mathbb{R}_{\geq 0} \rightarrow \mathbb{R}_{\geq 0}$ be locally integrable. Suppose there exists $\theta>0$ such that

$$
N:=\inf _{t \geq 0} \int_{t}^{t+\theta} p(s) d s>0 .
$$

Then, the system

$$
\dot{w}=-p(t) \varphi(w),
$$

has the following properties. 
a) For every $t_{0} \geq 0$ and $w_{0} \geq 0$, there exists a unique forward-in-time solution $w_{t_{0}, w_{0}}:\left[t_{0}, \infty\right) \rightarrow \mathbb{R}_{\geq 0}$ of (22) such that $w_{t_{0}, w_{0}}\left(t_{0}\right)=w_{0}$.

b) There exists $\beta \in \mathcal{K} \mathcal{L}$ such that

$$
w_{t_{0}, w_{0}}(t) \leq \beta\left(w_{0}, t-t_{0}\right) \quad \forall t \geq t_{0}, w_{0} \geq 0
$$

Proof. a) Consider the continuous and increasing function $F:(0, \infty) \rightarrow(a, b)$

$$
F(r)=\int_{1}^{r} \frac{d s}{\varphi(s)}
$$

with $a=\lim _{r \rightarrow 0^{+}} F(r)$ and $b=\lim _{r \rightarrow \infty} F(r) . F$ is bijective, and therefore its inverse $F^{-1}:(a, b) \rightarrow(0, \infty)$ is continuous and increasing.

Let $w$ be a solution of (22) such that $w\left(t_{0}\right)=w_{0}$. Since $w$ is nonincreasing and bounded from below, there is no finite escape time $t \geq t_{0}$ and therefore $w$ is defined on $\left[t_{0}, \infty\right)$. If $w(t)>0$ on an interval $\left[t_{0}, t_{1}\right)$, then a simple calculation shows that $w(t)=F^{-1}\left(F\left(w_{0}\right)-\int_{t_{0}}^{t} p(s) d s\right)$ for all $t \in\left[t_{0}, t_{1}\right)$. If for some $t_{1} \geq t_{0}$, $w\left(t_{1}\right)=0$, then the facts that $0 \leq w(t)$ for all $t \geq t_{1}$ and that $w$ is nonincreasing imply that $w(t)=0$ for all $t \geq t_{1}$. This shows the uniqueness forward-in-time of the solutions of (22).

b) It suffices to show that the nonnegative solutions of (22) satisfies (ii) and (ii). Let $t_{0} \geq 0, w_{0} \geq 0$ and $w_{t_{0}, w_{0}}:\left[t_{0}, \infty\right) \rightarrow \mathbb{R}_{\geq 0}$ be the unique solution of (22) such that $w\left(t_{0}\right)=w_{0}$. Since $w_{t_{0}, w_{0}}$ is nonincreasing, we have that $w_{t_{0}, w_{0}}(t) \leq w_{0}$. In consequence (ii) holds with $\alpha(r) \equiv r$.

As for (ii), let $0<\varepsilon \leq R$. Let $s \geq 0$ be so that $F^{-1}(F(R)-s)=\varepsilon$, pick $m_{0} \in \mathbb{N}$ so that $m_{0} N>s$, where $N$ is the constant appearing in (21), and define $T=m_{0} \theta$. Let $t_{0} \geq 0$ and $w_{0} \geq 0$. We claim that there is a $t_{0} \leq t_{1} \leq t_{0}+T$ such that $w_{t_{0}, w_{0}}\left(t_{1}\right) \leq \varepsilon$. If $w_{t_{0}, w_{0}}(t)>\varepsilon$ for all $t \in\left[t_{0}, t_{0}+T\right]$, then $w_{t_{0}, w_{0}}(t)=F^{-1}\left(F\left(w_{0}\right)-\int_{t_{0}}^{t_{0}+T} p(s) d s\right) \leq F^{-1}(F(R)-s)=\varepsilon$, since $\int_{t_{0}}^{t_{0}+T} p(s) d s \geq m N \geq s$, which is absurd. Then, since $w_{t_{0}, w_{0}}$ is nonincreasing, it follows that $w_{t_{0}, w_{0}}(t) \leq \varepsilon$ for all $t \geq t_{1}$.

Lemma 4.7. Let $\varphi, \psi \in \mathcal{P}$ and $p: \mathbb{R}_{\geq 0} \rightarrow \mathbb{R}_{\geq 0}$ be locally integrable. Then, maximally defined solutions of the comparison system (17) are forward complete. Moreover, if $z \in \mathcal{C}\left(t_{0}, z_{0}, \gamma\right)$ with $t_{0} \geq 0, z_{0} \geq 0$ and $\gamma \in \Gamma$ satisfies $z(s)=0$ for some $s \geq t_{0}$, then $z(t)=0$ for all $t \geq s$.

Proof. Let $z \in \mathcal{C}\left(t_{0}, z_{0}, \gamma\right)$ with $t_{0} \geq 0, z_{0} \geq 0$ and $\gamma \in \Gamma$. Suppose that $\left[t_{0}, T_{z}\right)$, with $T_{z}<\infty$ is the maximal interval of definition of $z$. Since $z$ is nonincreasing between consecutive impulse times and bounded from below, there exists $z\left(T_{z}^{-}\right)$. If $T_{z} \notin \gamma$, then there exists $\delta>0$ so that $\left[T_{z}, T_{z}+\delta\right) \cap \gamma=\emptyset$. Define $z^{*}(t)=z(t)$ if $t \in\left[t_{0}, T_{z}\right)$ and $z^{*}(t)=w^{*}(t)$ for $t \in\left[T_{z}, T_{z}+\delta\right)$, where $w^{*}$ is any solution of (22) such that $w^{*}\left(T_{z}\right)=z\left(T_{z}^{-}\right)$. If $T_{z} \in \Gamma$, take $\delta>0$ so that $\left(T_{z}, T_{z}+\delta\right) \cap \gamma=\emptyset$, and define $z^{*}$ as before, but with $w^{*}\left(T_{z}\right)=\psi\left(z\left(T_{z}^{-}\right)\right)$. In both cases $z^{*}$ is a proper extension of $z$ which is solution of (17), which is absurd. This show that $T_{z}=+\infty$. Suppose that $z(s)=0$ for some $s \geq t_{0}$. If $s_{0}=s$ and $s_{1}<s_{2}<\cdots$ are the impulse times in $\gamma \cap(s, \infty)$, we have that $z(t)=0$ for all $t \in\left[s_{0}, s_{1}\right)$ since $z$ is nonincreasing and nonnegative. Taking into account that $0 \leq z\left(s_{1}\right) \leq \psi\left(z\left(s_{1}^{-}\right)\right)=\psi(0)=0$, it follows that $z(t)=0$ for all $t \in\left[s_{1}, s_{2}\right)$. By proceeding in this way, it follows that $z(t)=0$ for all $t \geq s_{0}$.

Proof (Proposition 4.3). Let $\delta=N-M>0$.

(a) Let $M>0$ and $\mathcal{S} 匹 \overline{\mathcal{S}}\left(\frac{1}{\theta}\right)$. By (6) and Definition 3.3, for each $\varepsilon>0$ there is a $T(\varepsilon)>0$ such that

$$
\frac{n_{(s, s+t]}^{\gamma}}{t} \leq \frac{1}{\theta}+\varepsilon \quad \forall t \geq T(\varepsilon), \forall s \geq 0, \forall \gamma \in \mathcal{S} .
$$

Let $\varepsilon_{0}=\frac{\delta}{2 \theta M}$. Pick $m_{0} \in \mathbb{N}$ such that $m_{0} \theta=: T_{0} \geq T\left(\varepsilon_{0}\right)$ and let $k_{0}=\left\lfloor\left(\frac{1}{\theta}+\varepsilon_{0}\right) T_{0}\right\rfloor$. Then, $n_{\left(s, s+T_{0}\right]}^{\gamma} \leq k_{0}$ for all $s \geq 0$ and all $\gamma \in \mathcal{S}$. Pick any $\bar{\psi} \in \mathcal{K}_{\infty}$ satisfying $\max \{\psi$, id $\} \leq \bar{\psi}$, and define $\eta \in \mathcal{K}_{\infty}$ as $\eta=\bar{\psi} \bar{k}_{0}$, i.e.

$$
\eta:=\underbrace{\bar{\psi} \circ \cdots \circ \bar{\psi}}_{k_{0} \text { times }} .
$$


Note that if $k \in \mathbb{N}_{0}$ and $k \leq k_{0}$, then $11 \bar{\psi}^{k} \leq \eta$.

Let $t_{0} \geq 0, z_{0} \geq 0, \gamma \in \mathcal{S}$ and $z \in \mathcal{C}\left(t_{0}, z_{0}, \gamma\right)$. Due to Lemma 4.7, $z$ is defined for all $t \geq t_{0}$ and if $z(s)=0$ for some $s \geq t_{0}$ then $z(t)=0$ for all $t \geq s$. Taking into account that $z$ is nonincreasing between consecutive impulse times, that $\psi\left(z\left(t^{-}\right)\right) \leq \bar{\psi}\left(z\left(t^{-}\right)\right)$at each $t \in \gamma$, and that for all $t \in\left[t_{0}, t_{0}+T_{0}\right], k=n_{\left(t_{0}, t\right]}^{\gamma} \leq k_{0}$, it follows that $z(t) \leq \bar{\psi}^{k}\left(z_{0}\right) \leq \eta\left(z_{0}\right)$ for all $t \in\left[t_{0}, t_{0}+T_{0}\right]$; hence,

$$
\sup _{t_{0} \leq t \leq t_{0}+T_{0}} z(t) \leq \eta\left(z_{0}\right) .
$$

If $\gamma \cap\left(t_{0}, t_{0}+T_{0}\right]=\emptyset$, then $\dot{z}(t) \leq-p(t) \varphi(z(t))$ from (17a) implies that

$$
\int_{z\left(t_{0}+T_{0}\right)}^{z\left(t_{0}\right)} \frac{d s}{\varphi(s)} \geq \int_{t_{0}}^{t_{0}+T_{0}} p(t) d t \geq m_{0} N .
$$

If $\gamma \cap\left(t_{0}, t_{0}+T_{0}\right] \neq \emptyset$, consider the increasing sequence $\left\{t_{i}\right\}_{i=1}^{k}:=\gamma \cap\left(t_{0}, t_{0}+T_{0}\right]$. Note that $k \leq k_{0}$. Suppose that $z\left(t_{0}+T_{0}\right)>0$. Then $z(t)>0$ for all $t \in\left[t_{0}, t_{0}+T_{0}\right]$ due to Lemma 4.7. From (17), (14) and the definitions of $\varepsilon_{0}, m_{0}$ and $T_{0}$ it follows that

$$
\begin{aligned}
\int_{z\left(t_{0}+T_{0}\right)}^{z\left(t_{0}\right)} \frac{d s}{\varphi(s)} & =\sum_{i=0}^{k-1} \int_{z\left(t_{i+1}\right)}^{z\left(t_{i}\right)} \frac{d s}{\varphi(s)}+\int_{z\left(t_{0}+T_{0}\right)}^{z\left(t_{k}\right)} \frac{d s}{\varphi(s)}=\sum_{i=0}^{k-1}\left(\int_{z\left(t_{i+1}^{-}\right)}^{z\left(t_{i}\right)} \frac{d s}{\varphi(s)}+\int_{z\left(t_{i+1}\right)}^{z\left(t_{i+1}^{-}\right)} \frac{d s}{\varphi(s)}\right)+\int_{z\left(t_{0}+T_{0}\right)}^{z\left(t_{k}\right)} \frac{d s}{\varphi(s)} \\
& \geq \sum_{i=0}^{k-1} \int_{z\left(t_{i+1}^{-}\right)}^{z\left(t_{i}\right)} \frac{d s}{\varphi(s)}+\int_{z\left(t_{0}+T_{0}\right)}^{z\left(t_{k}\right)} \frac{d s}{\varphi(s)}+\sum_{i=1}^{k} \int_{\psi\left(z\left(t_{i}^{-}\right)\right)}^{z\left(t_{i}^{-}\right)} \frac{d s}{\varphi(s)} \geq \int_{t_{0}}^{t_{0}+T_{0}} p(t) d t-k M \\
& \geq m_{0} N-k M \geq m_{0} N-k_{0} M \geq m_{0} N-\left(\frac{1}{\theta}+\varepsilon_{0}\right) T_{0}=\frac{m_{0} \delta}{2}>0,
\end{aligned}
$$

where the first term in the first inequality in (28) follows from consideration of (17a). Taking into account (27) and (29) it follows that if $z\left(t_{0}+T_{0}\right)>0$ then

$$
\int_{z\left(t_{0}+T_{0}\right)}^{z\left(t_{0}\right)} \frac{d s}{\varphi(s)} \geq \frac{m_{0} \delta}{2}
$$

and, in consequence, $z\left(t_{0}+T_{0}\right)<z\left(t_{0}\right)$. Repeating the preceding reasoning on each of the intervals $\left[t_{0}+\right.$ $\left.(\ell-1) T_{0}, t_{0}+\ell T_{0}\right]$, with $\ell \in \mathbb{N}$, we have that $0<z\left(t_{0}+\ell T_{0}\right)<z\left(t_{0}+(\ell-1) T_{0}\right)$ or $z\left(t_{0}+\ell T_{0}\right)=0$ and that $\sup _{t_{0}+(\ell-1) T_{0} \leq t \leq t_{0}+\ell T_{0}} z(t) \leq \eta\left(z\left(t_{0}+(\ell-1) T_{0}\right)\right)$. Thus $z(t) \leq \eta\left(z_{0}\right)$ for all $t \geq t_{0}$ and the comparison system (17) is GUS according to (ii).

Next, let $0<\varepsilon \leq R$. Set $\bar{\varepsilon}:=\eta^{-1}(\varepsilon) \leq \varepsilon$ and pick $\ell \in \mathbb{N}$ such that

$$
\int_{\bar{\varepsilon}}^{R} \frac{d s}{\varphi(s)} \leq \frac{1}{2} m_{0} \ell \delta
$$

Let $z \in \mathcal{C}\left(t_{0}, z_{0}, \gamma\right)$ with $t_{0} \geq 0, z_{0} \geq 0$ and $\gamma \in \mathcal{S}$. Suppose that $z\left(t_{0}+\ell T_{0}\right)>0$. Then, from (30) we have

$$
\int_{z\left(t_{0}+\ell T_{0}\right)}^{R} \frac{d s}{\varphi(s)} \geq \int_{z\left(t_{0}+\ell T_{0}\right)}^{z\left(t_{0}\right)} \frac{d s}{\varphi(s)}=\sum_{r=0}^{\ell-1} \int_{z\left(t_{0}+(r+1) T_{0}\right)}^{z\left(t_{0}+r T_{0}\right)} \frac{d s}{\varphi(s)} \geq \frac{1}{2} m_{0} \ell \delta \geq \int_{\bar{\varepsilon}}^{R} \frac{d s}{\varphi(s)},
$$

\footnotetext{
${ }^{1}$ We define $\bar{\psi}^{0}=$ id.
} 
which implies that $z\left(t_{0}+\ell T_{0}\right) \leq \bar{\varepsilon}$ and then that $z(t) \leq \eta(\bar{\varepsilon})=\varepsilon$ for all $t \geq t_{0}+\ell T_{0}$. If $z\left(t_{0}+\ell T_{0}\right)=0$ then $z(t)=0$ for all $t \geq t_{0}+\ell T_{0}$. Therefore, uniform attractivity according to (ii) follows with $T(\varepsilon, R)=\ell T_{0}$. We have thus established that the comparison system is weakly GUAS uniformly over $\mathcal{S}$. From Lemma 3.6 iii), then $\mathcal{S}$ is UIB. Since the comparison system (17) is weakly GUAS uniformly over $\mathcal{S}$ and $\mathcal{S}$ is UIB, Lemma 4.5 that (17) is strongly GUAS uniformly over $\mathcal{S}$.

b) Let $M=0$. From (14), then $\psi(r) \leq r$ for all $r \geq 0$. Then, the result of Lemma 4.6 implies that the comparison system is weakly GUAS uniformly over $\Gamma$. To see this, let $z \in \mathcal{C}\left(t_{0}, z_{0}, \gamma\right)$, with $t_{0} \geq 0, z_{0} \geq 0$ and $\gamma \in \Gamma$. Applying well-known comparison results for ordinary differential equations, using in addition the fact that $\psi(r) \leq r$ and that, due to the uniqueness of the solutions of (22), $w_{t_{0}, z_{1}} \leq w_{t_{0}, z_{0}}$ if $0 \leq z_{1} \leq z_{0}$, it follows that $z(t) \leq w_{t_{0}, z_{0}}(t)$ for all $t \geq t_{0}$. In consequence, $z(t) \leq \beta\left(z_{0}, t-t_{0}\right)$ for all $t \geq t_{0}$, where $\beta \in \mathcal{K} \mathcal{L}$ is given by Lemma 4.6. Applying Lemma 4.5, then the comparison system is strongly GUAS uniformly over $\mathcal{S}$ for any UIB family $\mathcal{S} \subset \Gamma$.

C) Let $M<0<N$. From (14), then $0<\psi(r)<r$ for all $r>0$. Define $\bar{\psi}(r)=\max _{0 \leq s \leq r} \psi(s)$. Then $\bar{\psi}$ is continuous and nondecreasing, $0<\bar{\psi}(r)<r$ for all $r>0$ and $\bar{\psi}(0)=0$. Consider the following differential/difference inclusion system

$$
\begin{array}{llrl}
\dot{z}(t) & \in(-\infty,-p(t) \varphi(z(t))], & t \notin \gamma, \\
z(t) \in\left[0, \bar{\psi}\left(z\left(t^{-}\right)\right)\right], & t \in \gamma .
\end{array}
$$

We have that (17) is strongly GUAS uniformly over $\mathcal{S}$ if (33) is, since every solution of (17) is also a solution of (36). Due to Remark 3 in Mancilla-Aguilar and Haimovich (2019), for checking that (33) is strongly GUAS over $\Gamma$, it suffices to only consider the solutions of the impulsive system

$$
\begin{aligned}
& \dot{z}(t)=-p(t) \varphi(z(t)), \quad t \notin \gamma, \\
& z(t)=\bar{\psi}\left(z\left(t^{-}\right)\right), \quad \quad t \in \gamma,
\end{aligned}
$$

corresponding to initial times $t_{0} \geq 0$, initial conditions $z_{0} \geq 0$ and $\gamma \in \Gamma$. Consider the difference equation

$$
w_{k+1}=\bar{\psi}\left(w_{k}\right), \quad w_{0} \geq 0 .
$$

Since $\bar{\psi}(r)<r$ for all $r>0$, the discrete-time system is GUAS and there must exist $\beta_{d} \in \mathcal{K} \mathcal{L}$ such that every solution of this difference equation satisfies $w_{k} \leq \beta_{d}\left(w_{0}, k\right)$ for all $k \in \mathbb{N}_{0}$.

Let $z$ be a solution of (34) with $\gamma \in \Gamma$ such that $z\left(t_{0}\right)=z_{0}$, with $t_{0} \geq 0 z_{0} \geq 0$. Let $t>t_{0}$ and $k=n_{\left(t_{0}, t\right]}^{\gamma}$. Suppose that $k \geq 1$ and let $t_{0}<t_{1}<\cdots<t_{k} \leq t$ be all the impulse times within $\gamma \cap\left(t_{0}, t\right]$. Define $z_{j}=z\left(t_{j}\right)$ for $j=1, \ldots, k$. Since $z$ is nonincreasing between impulse times and $\bar{\psi}$ is nondecreasing, it can be proved by induction on $j$ that $z_{j} \leq w_{j}$ for all $j=0, \ldots, k$, where $\left\{w_{j}\right\}_{j=0}^{\infty}$ is the solution of (35) with $w_{0}=z_{0}$. Taking into account that $z(t) \leq z\left(t_{k}\right)=z_{k}$ and that $w_{k} \leq \beta_{d}\left(z_{0}, k\right)$, it follows that $z(t) \leq \beta_{d}\left(z_{0}, k\right)$. By the forward-in-time uniqueness of the solutions of (22) we have that $w_{t_{0}, \zeta}(s) \leq w_{t_{0}, \zeta^{*}}(s)$ for all $s \geq t_{0}$ if $0 \leq \zeta \leq \zeta^{*}$ and that $w_{t_{0}^{*}, \zeta^{*}}(t)=w_{t_{0}, \zeta}(t)$ for all $t \geq t_{0}^{*}$ if $\zeta^{*}=w_{t_{0}, \zeta}\left(t_{0}^{*}\right)$. Then, taking into account the latter and that $w_{t_{j}, z_{j}}\left(t_{j+1}\right) \geq z_{j+1}$ for all $j=0, \ldots, k-1$, it follows that $z_{k} \leq w_{t_{0}, z_{0}}\left(t_{k}\right)$ and then that $z(t) \leq w_{t_{0}, z_{0}}(t) \leq \beta\left(z_{0}, t-t_{0}\right)$. When $k=0$, we have that $z(t) \leq z_{0} \leq \beta_{d}\left(z_{0}, 0\right)$ and that $z(t)=w_{t_{0}, z_{0}}(t) \leq \beta\left(z_{0}, t-t_{0}\right)$. Since $k=n_{\left(t_{0}, t\right]}^{\gamma}$, we then have that

$$
z(t) \leq \min \left\{\beta\left(z_{0}, t-t_{0}\right), \beta_{d}\left(z_{0}, n_{\left(t_{0}, t\right]}^{\gamma}\right)\right\} \quad \forall t \geq t_{0} .
$$

By considering $\beta^{*}=\max \left\{\beta, \beta_{d}\right\} \in \mathcal{K} \mathcal{L}$ it follows that for all $t \geq t_{0}$

$$
z(t) \leq \min \left\{\beta^{*}\left(z_{0}, t-t_{0}\right), \beta^{*}\left(z_{0}, n_{\left(t_{0}, t\right]}^{\gamma}\right)\right\} \leq \beta^{*}\left(z_{0}, \max \left\{t-t_{0}, n_{\left(t_{0}, t\right]}^{\gamma}\right\}\right) .
$$

Since for every $a, b \in \mathbb{R}_{\geq 0}$ it happens that $\max \{a, b\} \geq(a+b) / 2$, and given that $\beta^{*} \in \mathcal{K} \mathcal{L}$, then for all $t \geq t_{0}$,

$$
\beta^{*}\left(z_{0}, \max \left\{t-t_{0}, n_{\left(t_{0}, t\right]}^{\gamma}\right\}\right) \leq \beta^{*}\left(z_{0}, \frac{t-t_{0}+n_{\left(t_{0}, t\right]}^{\gamma}}{2}\right)
$$

Since $\beta^{*}(\cdot, \cdot / 2) \in \mathcal{K} \mathcal{L}$, this completes the proof. 
Proof (Proposition 4.4). According to Lemma 6.2b) in Mancilla-Aguilar and Haimovich (2019), there exists $\psi^{*} \in \mathcal{K}_{\infty}$ such that $\psi \leq \psi^{*}$ and (15) holds with $\psi^{*}$ in place of $\psi$. Consider the comparison system

$$
\begin{array}{llrl}
\dot{z}(t) & \in(-\infty,-p(t) \varphi(z(t))], & & t \notin \gamma, \\
z(t) & \in\left[0, \psi^{*}\left(z\left(t^{-}\right)\right)\right], & t \in \gamma .
\end{array}
$$

We have that (17) is strongly GUAS uniformly over $\mathcal{S}$ if (36) is, since every solution of (17) is also a solution of (36). Due to Remark 3 in Mancilla-Aguilar and Haimovich (2019), for checking that (36) is strongly GUAS over $\mathcal{S}$, it suffices to only consider the solutions of the impulsive system

$$
\begin{aligned}
& \dot{z}(t)=-p(t) \varphi(z(t)), \quad t \notin \gamma, \\
& z(t)=\psi^{*}\left(z\left(t^{-}\right)\right), \quad t \in \gamma,
\end{aligned}
$$

corresponding to initial times $t_{0} \geq 0$, initial conditions $z_{0} \geq 0$ and $\gamma \in \mathcal{S}$. From the proof of Theorem 5.2 in Mancilla-Aguilar and Haimovich (2019), it follows that the continuous function $F:(0, \infty) \rightarrow \mathbb{R}$

$$
F(r)=\int_{1}^{r} \frac{d s}{-\varphi(s)}
$$

is bijective, and that $w_{t_{0}, w_{0}}(t)=F^{-1}\left(F\left(w_{0}\right)+\int_{t_{0}}^{t} p(s) d s\right), t \geq t_{0}$, is the unique forward-in-time solution of the initial value problem $\dot{w}=-p(t) \varphi(w), w\left(t_{0}\right)=w_{0}$, corresponding to $t_{0} \geq 0$ and $w_{0}>0$. It also holds that this initial value problem has a unique forward-in-time solution for $t_{0} \geq 0$ and $w_{0}=0$, which is the identically zero function. The latter implies that the maximal solutions of (37) corresponding to nonnegative initial times $t_{0}$ and initial conditions $w_{0}$ are unique and forward complete. Define

$$
M:=\inf _{a>0} \int_{\psi^{*}(a)}^{a} \frac{d s}{-\varphi(s)}, \quad N:=\sup _{t \geq 0} \int_{t}^{t+\theta} p(s) d s .
$$

Note that $M>N \geq 0$. Set $\delta=M-N>0$ and $\varepsilon_{0}=\frac{\delta}{2 \theta M}$. Since $\mathcal{S} 匹 \underline{\mathcal{S}}^{u}\left(\frac{1}{\theta}\right)$, there exists $T\left(\varepsilon_{0}\right)>0$ such that $\frac{n_{(s, s+t]}^{\gamma}}{t} \geq \frac{1}{\theta}-\varepsilon_{0}$ for all $t \geq T\left(\varepsilon_{0}\right)$, all $s \geq 0$ and all $\gamma \in \mathcal{S}$. Pick $m_{0} \in \mathbb{N}$ so that $T_{0}:=m_{0} \theta \geq T\left(\varepsilon_{0}\right)$ and let $k_{0}:={ }^{t}\left\lceil\left(\frac{1}{\theta}-\varepsilon_{0}\right) m_{0} \theta\right\rceil$. Note that for any $\gamma \in \mathcal{S}$ the $k_{0}$-th impulse time after any $t \geq 0$ must belong to the interval $\left[t, t+T_{0}\right]$. Define $\nu(r)=F^{-1}\left(F(r)+m_{0} N\right)$ if $r>0$ and $\nu(0)=0$. We have that $\nu \in \mathcal{K}_{\infty}$.

Let $z$ be a solution of (37) corresponding to $t_{0} \geq 0, z_{0}>0$ and $\gamma \in \Gamma$. Since $z$ is nondecreasing between impuse times, and $\psi^{*} \in \mathcal{K}_{\infty}$, it follows that $z(t)>0$ for all $t \geq t_{0}$. Let $w_{t_{0}, z_{0}}$ as above, then, due to the uniqueness of the solutions of the initial value problems $\dot{w}=-p(t) \varphi(w), w\left(t_{0}\right)=w_{0}$ and the fact that $\psi^{*}(r)<r$ for all $r>0$, since $M>0$, we have that $z(t) \leq w_{t_{0}, z_{0}}(t)=F^{-1}\left(F\left(w_{0}\right)+\int_{t_{0}}^{t} p(s) d s\right)$ for all $t \geq t_{0}$. In particular, taking into account that $\int_{t_{0}}^{t_{0}+T_{0}} p(s) d s \leq m_{0} N$, it follows that

$$
z(t) \leq \nu\left(z_{0}\right) \quad \forall t \in\left[t_{0}, t_{0}+T_{0}\right]
$$

Let $t_{1}<t_{2}<\cdots$ be the sequence of impulse times after time $t_{0}$, that is $\left\{t_{k}\right\}_{k=1}^{\infty}=\gamma \cap\left(t_{0}, \infty\right)$. Note that necessarily $t_{k_{0}} \in\left[t_{0}, t_{0}+T_{0}\right]$. From (37) it follows that

$$
\begin{aligned}
\int_{z\left(t_{0}\right)}^{z\left(t_{k_{0}}\right)} \frac{d s}{-\varphi(s)} & =\sum_{k=0}^{k_{0}-1} \int_{z\left(t_{k}\right)}^{z\left(t_{k+1}^{-}\right)} \frac{d s}{-\varphi(s)}+\sum_{k=1}^{k_{0}} \int_{z\left(t_{k}^{-}\right)}^{z\left(t_{k}\right)} \frac{d s}{-\varphi(s)}=\sum_{k=0}^{k_{0}-1} \int_{z\left(t_{k}\right)}^{z\left(t_{k+1}^{-}\right)} \frac{d s}{-\varphi(s)}+\sum_{k=1}^{k_{0}} \int_{z\left(t_{k}\right)^{-}}^{\psi^{*}\left(z\left(t_{k}\right)\right)} \frac{d s}{-\varphi(s)} \\
& \leq \int_{t_{0}}^{t_{k_{0}}} p(s) d s-k_{0} M \leq m_{0} N-\left(\frac{1}{\theta}-\varepsilon_{0}\right) m_{0} \theta M \leq-\frac{1}{2} m_{0} \delta .
\end{aligned}
$$

In consequence $z\left(t_{k_{0}}\right)<z\left(t_{0}\right)$. Taking into account (39), (40), and applying the preceding reasoning with $t_{\ell k_{0}}$ as $t_{0}$ and $t_{(\ell+1) k_{0}}$ as $t_{k_{0}}$, we have that $\left\{z\left(t_{\ell k_{0}}\right)\right\}_{\ell=0}^{\infty}$ is a decreasing sequence, and that for all $t \in\left[t_{\ell k_{0}}, t_{(\ell+1) k_{0}}\right]$, 
we have that $z(t) \leq \nu\left(z\left(t_{\ell k_{0}}\right)\right) \leq \nu\left(z_{0}\right)$ since $\left[t_{\ell k_{0}}, t_{(\ell+1) k_{0}}\right] \subset\left[t_{\ell k_{0}}, t_{\ell k_{0}}+T_{0}\right]$. Taking into account that (40) holds with $t_{\ell k_{0}}$ and $t_{(\ell+1) k_{0}}$ in place of $t_{0}$ and $t_{k_{0}}$, it follows that

$$
\int_{z\left(t_{0}\right)}^{z\left(t_{\ell k_{0}}\right)} \frac{d s}{-\varphi(s)} \leq-\frac{1}{2} \ell m_{0} \delta
$$

and therefore $z\left(t_{\ell k_{0}}\right) \leq F^{-1}\left(F\left(z\left(t_{0}\right)\right)-\frac{1}{2} \ell m_{0} \delta\right)$. Define

$$
\beta_{1}(r, s):= \begin{cases}F^{-1}\left(F(r)-\frac{1}{2} m_{0} \delta s\right) & \text { if } r>0 \text { and } s \geq 0, \\ 0 & \text { if } r=0 \text { and } s \geq 0,\end{cases}
$$

and let $\beta_{2}=\nu \circ \beta_{1}$. Then $\beta_{2} \in \mathcal{K} \mathcal{L}$ and $z(t) \leq \beta_{2}\left(z\left(t_{0}\right), \ell\right)$ if $t \in\left[t_{\ell k_{0}}, t_{(\ell+1) k_{0}}\right)$. Note that for such a value of $t, \ell k_{0} \leq n_{\left(t_{0}, t\right]}^{\gamma}<(\ell+1) k_{0}$ and then $\ell=\left\lfloor n_{\left(t_{0}, t\right]}^{\gamma} / k_{0}\right\rfloor$. In consequence,

$$
z(t) \leq \beta_{2}\left(z\left(t_{0}\right),\left\lfloor n_{\left(t_{0}, t\right]}^{\gamma} / k_{0}\right\rfloor\right), \quad \forall t \geq t_{0} .
$$

Define $\beta_{3}: \mathbb{R}_{\geq 0} \times \mathbb{R}_{\geq 0} \rightarrow \mathbb{R}_{\geq 0}$ via

$$
\beta_{3}(r, s)= \begin{cases}\left(2-\frac{s}{k_{0}}\right) \beta_{2}(r, 0) & 0 \leq s<k_{0}, r \geq 0 \\ \beta_{2}\left(r, \frac{s}{k_{0}}-1\right), & s \geq k_{0}, r \geq 0\end{cases}
$$

Then $\beta_{3} \in \mathcal{K} \mathcal{L}$ and

$$
z(t) \leq \beta_{3}\left(z\left(t_{0}\right), n_{\left(t_{0}, t\right]}^{\gamma}\right), \quad \forall t \geq t_{0} .
$$

Since $n_{\left(t_{0}, t\right]}^{\gamma} \geq\left(\frac{1}{\theta}-\varepsilon_{0}\right)\left(t-t_{0}\right)$ if $t-t_{0} \geq T_{0}$, it follows that

$$
\frac{1}{2} n_{\left(t_{0}, t\right]}^{\gamma} \geq \max \left\{\kappa\left(t-t_{0}\right)-\kappa T_{0}, 0\right\} \quad \forall t \geq t_{0},
$$

where $\kappa=\frac{1}{2}\left(\frac{1}{\theta}-\varepsilon_{0}\right)$. The combination of (43) with (44) yields that, for all $t \geq t_{0}$,

$$
z(t) \leq \beta_{3}\left(z\left(t_{0}\right), \frac{n_{\left(t_{0}, t\right]}^{\gamma}}{2}+\max \left\{\kappa\left(t-t_{0}\right)-\kappa T_{0}, 0\right\}\right) .
$$

From the latter and proceeding as in the last part of the proof of Theorem 5.2 in Mancilla-Aguilar and Haimovich (2019) it follows that there exits $\beta \in \mathcal{K} \mathcal{L}$ so that

$$
z(t) \leq \beta\left(z\left(t_{0}\right), t-t_{0}+n_{\left(t_{0}, t\right]}^{\gamma}\right) \quad \forall t \geq t_{0} .
$$

This establishes the required strong GUAS property.

\section{Examples}

\subsection{Destabilizing impulses}

Consider a scalar and single-input impulsive system of the form (11) with time-invariant flow and jump maps, defined as

$$
f(t, \xi, \mu)=\bar{f}(\xi, \mu)=\left\{\begin{array}{ll}
-\xi+\sqrt{2} \mu & \text { if }|\xi| \leq \sqrt{2} \\
-\frac{\xi^{3}}{2}+|\xi| \mu & \text { if }|\xi|>\sqrt{2}
\end{array} \quad g(t, \xi, \mu)=\bar{g}(\xi, \mu)= \begin{cases}2 \sqrt{2} \xi^{2} \mu & \text { if }|\xi| \leq \sqrt{2} \\
\xi^{3} & \text { if }|\xi|>\sqrt{2}\end{cases}\right.
$$


Note that under zero input, the flow is stabilizing and the jumps are destabilizing. We would like to determine a class of impulse-time sequences over which this system is ISS in the standard sense. We thus consider $h^{o}(t, \xi)=h(t, \xi)=|\xi|$ and search for an ISS Lyapunov function candidate. Taking $V(t, \xi)=\bar{V}(\xi)=\xi^{2} / 2$, we compute

$$
D_{f}^{+} \bar{V}(\xi, \mu)=\left\{\begin{array}{ll}
-\xi^{2}+\sqrt{2} \xi \mu & \text { if }|\xi| \leq \sqrt{2}, \\
-\frac{\xi^{4}}{2}+\xi^{2} \mu & \text { if }|\xi|>\sqrt{2},
\end{array} \quad \bar{V}(\xi+\bar{g}(\xi, \mu))= \begin{cases}\left(\xi+2 \sqrt{2} \xi^{2} \mu\right)^{2} / 2 & \text { if }|\xi| \leq \sqrt{2} \\
\left(\xi+\xi^{3}\right)^{2} / 2 & \text { if }|\xi|>\sqrt{2}\end{cases}\right.
$$

If it happens that

$$
|\mu| \leq \eta(|\xi|):= \begin{cases}\frac{|\xi|}{2 \sqrt{2}} & \text { if }|\xi| \leq \sqrt{2}, \\ \frac{|\xi|^{2}}{4} & \text { if }|\xi|>\sqrt{2},\end{cases}
$$

then

$$
\begin{aligned}
D_{f}^{+} \bar{V}(\xi, \mu) & \leq \begin{cases}-\xi^{2} / 2=-\bar{V}(\xi) & \text { if }|\xi| \leq \sqrt{2}, \\
-\xi^{4} / 4=-\bar{V}(\xi)^{2} & \text { if }|\xi|>\sqrt{2},\end{cases} \\
\bar{V}(\xi+\bar{g}(\xi, \mu)) & \leq\left(|\xi|+|\xi|^{3}\right)^{2} / 2=\psi(\bar{V}(\xi)),
\end{aligned}
$$

Since $\eta \in \mathcal{K}_{\infty}$, we may define $\chi=\left(\eta^{-1}\right)^{2} / 2 \in \mathcal{K}_{\infty}$. Define also $\varphi, p: \mathbb{R}_{\geq 0} \rightarrow \mathbb{R}_{\geq 0}$ via

$$
\varphi(s):=\left\{\begin{array}{ll}
s & \text { if } s \leq 1, \\
s^{2} & \text { if } s>1,
\end{array} \quad p(s) \equiv 1 .\right.
$$

Also, we have $\bar{V}(\xi) \leq \chi(|\mu|)$ if and only if $|\xi| \leq \eta^{-1}(|\mu|)$. As a consequence, whenever $\bar{V}(\xi) \leq \chi(|\mu|)$ then

$$
\bar{V}(\xi+g(t, \xi, \mu)) \leq\left(\eta^{-1}(|\mu|)+\left[\eta^{-1}(|\mu|)\right]^{3}\right)^{2} / 2=: \pi(|\mu|) .
$$

It follows that $V$ is an ISS Lyapunov function candidate with $\chi, \pi, \varphi$ and $\psi$ as defined. To apply Theorem 4.1 we compute

$$
\int_{a}^{\psi(a)} \frac{d s}{\varphi(s)}= \begin{cases}\ln \left(\frac{\psi(a)}{a}\right) & \text { if } \psi(a) \leq 1 \\ \frac{1}{a}-\frac{1}{\psi(a)} & \text { if } a \geq 1 \\ \ln \left(\frac{1}{a}\right)+1-\frac{1}{\psi(a)} & \text { if } a<1<\psi(a) .\end{cases}
$$

and we obtain

$$
\begin{aligned}
& M=\sup _{a>0} \int_{a}^{\psi(a)} \frac{d s}{\varphi(s)}=\int_{0.5}^{\psi(0.5)} \frac{d s}{\varphi(s)} \approx 1.1931, \\
& N=\inf _{t \geq 0} \int_{t}^{t+\theta} p(s) d s=\theta .
\end{aligned}
$$

Application of Theorem 4.1 gives that the system is strongly ISS over any $\mathcal{S} \subset \Gamma$ such that $\mathcal{S} \backsim \overline{\mathcal{S}}(1 / \theta)$ whenever $\theta>M$.

\subsection{Stabilizing impulses}

We consider a scalar time-varying impulsive system with no inputs and flow and jump maps given by (1) with

$$
\begin{aligned}
& f(t, \xi, \mu)=\tanh (t) \tanh (\xi) \\
& g(t, \xi, \mu)= \begin{cases}-\xi+\xi^{3} / 2 & \text { if }|\xi| \leq 1 \\
-\xi+\xi^{1 / 3} / 2 & \text { if }|\xi|>1\end{cases}
\end{aligned}
$$


The flow map is a time-varying multiple of that in Example 1 of Feketa and Bajcinca (2019b) and the jump map coincides. We would like to know the largest class of impulse-time sequences over which the impulsive system is GUAS, according to Theorem 4.2. Since the system has no inputs, we take $m=0$ and consider $u \in \mathbb{R}^{0}$. Since standard GUAS is considered, we take $h^{o}(t, \xi)=h(t, \xi)=|\xi|$. Taking $V(t, \xi)=\bar{V}(\xi)=|\xi|$, it follows that $V$ is a $\left(h^{o}, h\right)$-ISS Lyapunov function candidate with

$$
p(t)=\tanh (t), \quad \psi(s)=-\tanh (s), \quad \psi(s)= \begin{cases}s^{3} / 2 & \text { if } 0 \leq s \leq 1, \\ s^{1 / 3} / 2 & \text { if } s>1,\end{cases}
$$

and arbitrary $\chi, \pi \in \mathcal{K}_{\infty}$. We have

$$
\sup _{t \geq 0} \int_{t}^{t+\theta} \tanh (s) d s=\theta
$$

and as in Example 1 of Feketa and Baicinca (2019b),

$$
\inf _{a>0} \int_{\psi(a)}^{a} \frac{d s}{-\varphi(s)}=\ln (1+e)-\frac{1}{2} \approx 0.81
$$

Also, (16) is satisfied. Theorem 4.2 ensures that the impulsive system is strongly $\left(h^{0}, h\right)$-ISS, hence in this case strongly GUAS, over any class $\mathcal{S} \subset \Gamma$ satisfying $\mathcal{S} 匹 \underline{\mathcal{S}}(1 / \theta)$ provided that $0<\theta<\ln (1+e)-\frac{1}{2}$. Therefore, strong GUAS is ensured over any uniform subset of the class of impulse-time sequences with i.f. eventually uniformly lower bounded by $1 / \theta$. For comparison with Example 1 of Feketa and Bajcinca

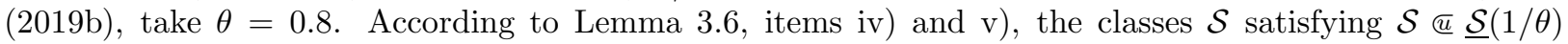
are much larger than what is covered by the results in Feketa and Baicinca (2019b). In particular, the impulsive system is GUAS over sequences with consecutive impulses much more separated than the value $3 / 2$ in Example 1 of Feketa and Bajcinca (2019b) provided that the i.f. eventually becomes uniformly lower bounded by $1 / \theta=1.25$. In addition, note that the results of Feketa and Bajcinca (2019b), by contrast to the current ones, only ensure standard, i.e. weak, GUAS that is not uniform w.r.t. initial time.

\section{Conclusions}

We have developed novel sufficient conditions for the weak or strong ISS of nonlinear time-varying impulsive systems with inputs, employing a two-measure framework. These conditions generalize, extend, and strengthen many existing results by ensuring ISS that holds uniformly over impulse-time sequences having eventually uniformly bounded impulse frequency. We show that the considered classes of impulsetime sequences are broader than most other sequence classes considered in the literature. In particular, sequences with fixed and average dwell times, as well as sequences where the impulse frequency achieves uniform convergence to a limit (superior or inferior) are all covered.

\section{Acknowledgments}

Work partially supported by Agencia Nacional de Promoción Científica y Tecnológica, Argentina, under grant PICT 2018-01385.

\section{References}

Cai, C., Teel, A. R., 2005. Results on input-to-state stability for hybrid systems. In: Proc. 44th IEEE Conf. on Decision and Control, Seville, Spain. pp. 5403-5408.

Cai, C., Teel, A. R., 2009. Characterizations of input-to-state stability for hybrid systems. Systems and Control Letters 58 (1), $47-53$.

Chatterjee, D., Liberzon, D., 2006. Stability analysis of deterministic and stochastic switched systems via a comparison principle and multiple Lyapunov functions. SIAM J. Control and Optimization 45 (1), 174-206. 
Dashkovskiy, S., Feketa, P., 2016. Input-to-state stability of impulsive systems with different jump maps. IFAC-PapersOnLine 49 (18), 1073 - 1078, 10th IFAC Symposium on Nonlinear Control Systems NOLCOS 2016.

Dashkovskiy, S., Feketa, P., 2017. Input-to-state stability of impulsive systems and their networks. Nonlinear Analysis: Hybrid Systems 26, $190-200$.

Dashkovskiy, S., Kosmykov, M., Mironchenko, A., Naujok, L., 2012. Stability of interconnected impulsive systems with and without time delays, using Lyapunov methods. Nonlinear Analysis: Hybrid Systems 6 (3), 899-915.

Dashkovskiy, S., Mironchenko, A., 2013. Input-to-state stability of nonlinear impulsive systems. SIAM Journal on Control and Optimization $51(3), 1962-1987$.

Feketa, P., Bajcinca, N., 2018. Stability of nonlinear impulsive differential equations with non-fixed moments of jumps. In: Proceedings of the 2018 European Control Conference (ECC), Limassol, Cyprus. pp. 900-905.

Feketa, P., Bajcinca, N., 2019a. Average dwell-time for impulsive control systems possessing ISS-Lyapunov function with nonlinear rates. In: 2019 18th European Control Conference (ECC). IEEE, pp. 3686-3691.

Feketa, P., Bajcinca, N., 2019b. On robustness of impulsive stabilization. Automatica 104, 48-56.

Feng, Y., Yu, J., Li, C., Huang, T., Che, H., 2017. Linear impulsive control system with impulse time windows. Journal of Vibration and Control 23 (1), 111-118.

Haimovich, H., Mancilla-Aguilar, J. L., 2018. A characterization of iISS for time-varying impulsive systems. In: Argentine Conference on Automatic Control (AADECA). Buenos Aires, Argentina, pp. 1-6.

Haimovich, H., Mancilla-Aguilar, J. L., 2019a. Nonrobustness of asymptotic stability of impulsive systems with inputs. Submitted to Automatica. Available at http://arxiv.org/abs/1910.07610.

Haimovich, H., Mancilla-Aguilar, J. L., 2019b. Strong ISS implies strong iISS for time-varying impulsive systems. Submitted to Automatica. Available at http://arxiv.org/abs/1909.00858.

Hale, J. K., 1980. Ordinary Differential Equations. Robert E. Krieger Publishing Company, Malabar, Florida.

Hespanha, J. P., Liberzon, D., Teel, A. R., 2005. On input-to-state stability of impulsive systems. In: Proc. of the 44th IEEE Conf. on Decision and Control and 2005 European Control Conf. pp. 3992-3997.

Hespanha, J. P., Liberzon, D., Teel, A. R., 2008. Lyapunov conditions for input-to-state stability of impulsive systems. Automatica 44 (11), 2735-2744.

Lakshmikantham, V., Bainov, D., Simeonov, P. S., 1989. Theory of impulsive differential equations. World Scientific.

Liberzon, D., Nešić, D., Teel, A. R., 2014. Lyapunov-based small-gain theorems for hybrid systems. IEEE Trans. on Automatic Control 59 (6), 1395-1410.

Lin, Y., Sontag, E. D., Wang, Y., 1996. A smooth converse Lyapunov theorem for robust stability. SIAM J. Control and Optimization 34 (1), 124-160.

Liu, J., Liu, X., Xie, W.-C., 2011. Input-to-state stability of impulsive and switching hybrid systems with time-delay. Automatica J. IFAC $47(5), 899-908$.

Liu, J., Liu, X., Xie, W.-C., 2012. Class-KL estimates and input-to-state stability analysis of impulsive switched systems. Systems \& Control Letters 61 (6), 738-746.

Mancilla-Aguilar, J. L., Haimovich, H., 2019. Uniform input-to-state stability for switched and time-varying impulsive systems. Submitted to IEEE TAC. Available at http://arxiv.org/abs/1904.03440.

Ning, C., He, Y., Wu, M., Zhou, S., 2018. Indefinite lyapunov functions for input-to-state stability of impulsive systems. Information Sciences 436, 343-351.

Peng, S., 2018. Lyapunov-Krasovskii-type criteria on ISS and iISS for impulsive time-varying delayed systems. IET Control Theory \& Applications 12 (11), 1649-1657.

Peng, S., Deng, F., Zhang, Y., 2018. A unified Razumikhin-type criterion on input-to-state stability of time-varying impulsive delayed systems. Systems \& Control Letters 116, 20-26.

Ren, W., Xiong, J., 2017. Stability analysis of impulsive stochastic nonlinear systems. IEEE Trans. Autom. Control 62 (9), 4791-4797.

Rivadeneira, P. S., Moog, C. H., 2015. Observability criteria for impulsive control systems with applications to biomedical engineering processes. Automatica 55, 125-131.

Rogovchenko, Y. V., 1997. Nonlinear impulse evolution systems and applications to population models. Journal of Mathematical Analysis and Applications 207 (2), 300-315.

Samoilenko, A., Perestyuk, N., 1987. Differential equations with impulse effect. Visca Skola, Kiev.

Samoilenko, A. M., Perestyuk, N. A., 1995. Impulsive differential equations. Vol. 14 of World Scientific Series on Nonlinear Science. World Scientific Publishing Co., Inc., River Edge, NJ. URL http://dx.doi.org/10.1142/9789812798664

Sontag, E. D., 1989. Smooth stabilization implies coprime factorization. IEEE Trans. on Automatic Control 34 (4), $435-443$.

Sontag, E. D., Wang, Y., 2000. Lyapunov characterizations of input to output stability. SIAM J. Control and Optimization 39 (1), 226-249.

Sun, X.-M., Wang, W., 2012. Integral input-to-state stability for hybrid delayed systems with unstable continuous dynamics. Automatica J. IFAC 48 (9), 2359-2364.

Tan, J., Li, C., Huang, T., Dec 2015. Stability of impulsive systems with time window via comparison method. International Journal of Control, Automation and Systems 13 (6), 1346-1350. URL https://doi.org/10.1007/s12555-014-0197-y

Tang, Y., Xing, X., Karimi, H. R., Kocarev, L., Kurths, J., 2015. Tracking control of networked multi-agent systems under new characterizations of impulses and its applications in robotic systems. IEEE Transactions on Industrial Electronics 63 (2), $1299-1307$.

Wu, X., Tang, Y., Zhang, W., 2016. Input-to-state stability of impulsive stochastic delayed systems under linear assumptions. 
Automatica 66, 195-204.

Yang, X., Peng, D., Lv, X., Li, X., 2019. Recent progress in impulsive control systems. Mathematics and Computers in Simulation 155, 244-268.

Yao, F., Qiu, L., Shen, H., 2014. On input-to-state stability of impulsive stochastic systems. J. Franklin Inst. 351 (9), $4636-4651$. 